This item was submitted to Loughborough's Research Repository by the author.

Items in Figshare are protected by copyright, with all rights reserved, unless otherwise indicated.

\title{
Effect of electron-phonon interaction range on lattice polaron dynamics: a continuous-time quantum Monte Carlo study
}

PLEASE CITE THE PUBLISHED VERSION

http://dx.doi.org/10.1103/PhysRevB.71.184310

\section{PUBLISHER}

(C) Americal Physical Society

\section{VERSION}

VoR (Version of Record)

\section{LICENCE}

CC BY-NC-ND 4.0

\section{REPOSITORY RECORD}

Spencer, Paul E., J.H. Samson, P.E. Kornilovitch, and A.S. Alexandrov. 2019. "Effect of Electron-phonon Interaction Range on Lattice Polaron Dynamics: A Continuous-time Quantum Monte Carlo Study". figshare. https://hdl.handle.net/2134/11613. 
This item was submitted to Loughborough's Institutional Repository (https://dspace.lboro.ac.uk/) by the author and is made available under the following Creative Commons Licence conditions.

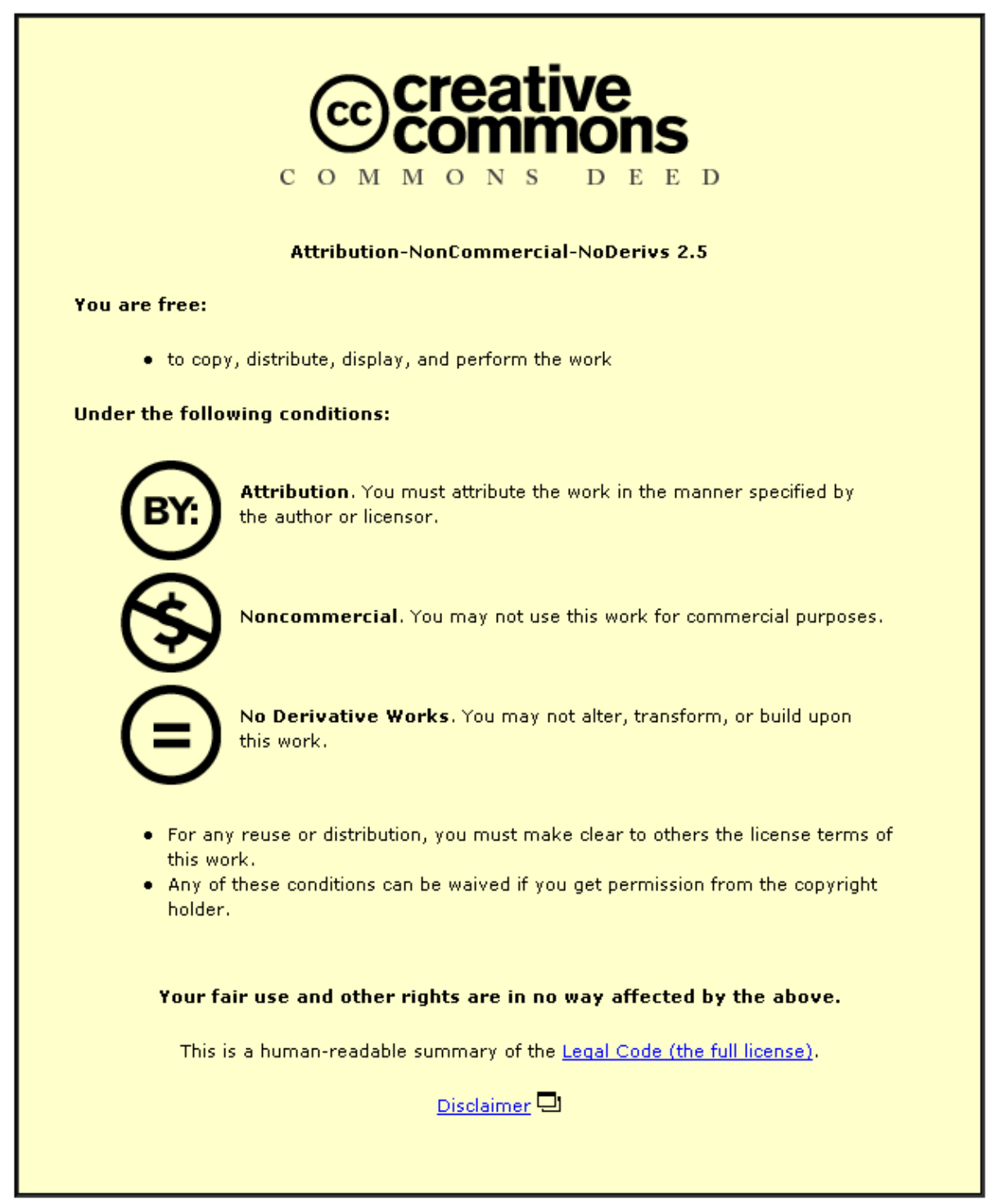

For the full text of this licence, please go to: http://creativecommons.org/licenses/by-nc-nd/2.5/ 


\title{
Effect of electron-phonon interaction range on lattice polaron dynamics: A continuous-time quantum Monte Carlo study
}

\author{
P. E. Spencer, ${ }^{1}$ J. H. Samson, ${ }^{1, *}$ P. E. Kornilovitch, ${ }^{2, \dagger}$ and A. S. Alexandrov ${ }^{1, \ddagger}$ \\ ${ }^{1}$ Department of Physics, Loughborough University, Loughborough LE11 3TU, United Kingdom \\ ${ }^{2}$ Hewlett-Packard Company, Mail Stop 321A, 1000 NE Circle Blvd, Corvallis, Oregon 97330, USA \\ (Received 9 July 2004; revised manuscript received 23 December 2004; published 31 May 2005)
}

\begin{abstract}
We present the numerically exact ground-state energy, effective mass, and isotope exponents of a onedimensional lattice polaron, valid for any range of electron-phonon interaction, applying a continuous-time quantum Monte Carlo (QMC) technique in a wide range of coupling strength and adiabatic ratio. The QMC method is free from any systematic finite-size and finite-time-step errors. We compare our numerically exact results with analytical weak-coupling theory and with the strong-coupling $1 / \lambda$ expansion. We show that the exact results agree well with the canonical Fröhlich and Holstein-Lang-Firsov theories in the weak and strong coupling limits, respectively, for any range of interaction. We find a strong dependence of the polaron dynamics on the range of interaction. An increased range of interaction has a similar effect to an increased (less adiabatic) phonon frequency: specifically, a reduction in the effective mass.
\end{abstract}

DOI: $10.1103 /$ PhysRevB.71.184310

PACS number(s): 71.38.-k, 02.70.Ss

\section{INTRODUCTION}

While qualitative features of polarons were well recognized a long time ago and have been described in several review papers and textbooks (see Refs. 1-4 for recent publications), there is renewed interest in quantitative studies owing to the overwhelming evidence for polaronic carriers in cuprates, fullerenes, and manganites (see, for example, Refs. $3-10)$. Under certain conditions ${ }^{11}$ the multipolaron system can be metallic but with polaronic carriers rather than bare electrons. There is a qualitative difference between the ordinary metal and the polaronic one. One can account for the electron-phonon (e-ph) interaction in simple metals by applying Migdal's theorem. ${ }^{12}$ The theorem shows that the contribution of diagrams with "crossing" phonon lines (so-called "vertex" corrections) is small if the parameter $\lambda \hbar \omega / E_{F}$ is small, where $\lambda$ is the dimensionless (BCS) $e$-ph coupling constant, $\omega$ is the characteristic phonon frequency, and $E_{F}$ is the Fermi energy. Neglecting the vertex corrections, Migdal calculated the renormalized electron mass as $m^{*}=m_{0}(1+\lambda)$ (near the Fermi level), ${ }^{12}$ where $m_{0}$ is the band mass in the absence of electron-phonon interaction, and Eliashberg ${ }^{13}$ extended Migdal's theory to describe the BCS superconducting state at intermediate values of $\lambda, \lambda \leqslant 1$. Later on many authors applied Migdal-Eliashberg theory with $\lambda$ much larger than 1 (see, for example, Ref. 14).

On the other hand, starting from the infinite coupling limit $\lambda=\infty$ and applying the inverse $(1 / \lambda)$ expansion technique ${ }^{15}$ one can show ${ }^{16-18}$ that the many-electron system collapses into the small polaron regime at $\lambda \sim 1$ almost independently of the adiabatic ratio $\hbar \omega / E_{F}$. This regime is beyond MigdalEliashberg theory, where the effective mass approximation is used and the electron bandwidth is infinite. It is a wellestablished theorem that a self-trapping crossover is analytical in the coupling strength, so that one could believe that the sum of all diagrams (including the vertex corrections) should produce the exact solution if the expansion converges. Indeed, results of QMC simulations based on summing the
Feynman diagrams ${ }^{19}$ provide the exact answer in the continuous (large polaron) model. On the other hand, the small polaron regime cannot be reached by summation of the standard Feynman-Dyson perturbation diagrams using a translation-invariant Green function $G\left(\mathbf{r}, \mathbf{r}^{\prime}, \tau\right)=G\left(\mathbf{r}-\mathbf{r}^{\prime}, \tau\right)$ with the Fourier transform $G(\mathbf{k}, \Omega)$ prior to solving the Dyson equations on a discrete lattice. This assumption excludes the possibility of local violation of the translational symmetry ${ }^{20}$ due to the lattice deformation in any order of the Feynman-Dyson perturbation theory similar to the absence of the anomalous (Bogoliubov) averages in any order of perturbation theory. ${ }^{12}$ One way to describe the formation of the lowest polaronic band is to introduce an infinitesimal translation-noninvariant potential, which should be set to zero only in the final solution obtained by the summation of Feynman diagrams for the Fourier transform $G\left(\mathbf{k}, \mathbf{k}^{\prime}, \Omega\right)$ of $G\left(\mathbf{r}, \mathbf{r}^{\prime}, \tau\right)$ rather than for $G(\mathbf{k}, \Omega) \cdot{ }^{17}$ As in the case of the off-diagonal superconducting order parameter, the offdiagonal terms of the Green function, in particular the Umklapp terms with $\mathbf{k}^{\prime}=\mathbf{k}+\mathbf{G}$, drive the system into a small polaron ground state at sufficiently large coupling. Setting the translation-noninvariant potential to zero in the solution of the equations of motion restores the translation symmetry but in a polaron band rather than in the bare electron band, which turns out to be an excited state. ${ }^{21}$ Alternatively, one can work with momentum eigenstates throughout the whole coupling region, but taking into account the finite-electron bandwidth (i.e., including Umklapp terms). In recent years many such numerical and analytical studies have confirmed the conclusion ${ }^{16}$ that the Migdal-Eliashberg theory breaks down at $\lambda \gtrsim 1$ (see Refs. 22-36, and references therein).

In ordinary metals, where the Migdal approximation is believed to be valid, the renormalized effective mass of electrons is independent of the ion mass $M$ because the electronphonon interaction constant $\lambda$ does not depend on $M$. However, when the $e$-ph interaction is sufficiently strong, the electrons form polarons dressed by lattice distortions, with an effective mass $m^{*}=m_{0} \exp \left(\gamma E_{p} / \hbar \omega\right)$. Here $E_{p}$ is the po- 
laron binding energy (or the polaron shift), and $\gamma$ is a numerical constant that depends on the radius of electronphonon interaction and is typically less than 1 . While $E_{p}$ in the above expression does not depend on the ion mass, the phonon frequency does. As a result, there is a large isotope effect on the carrier mass in polaronic conductors $\alpha_{m}$ $=(1 / 2) \ln \left(m^{*} / m\right),{ }^{37}$ in contrast to the zero isotope effect in ordinary metals. Such an effect was found experimentally in the cuprates ${ }^{10}$ and manganites. ${ }^{38}$ A recent high-resolution angle resolved photoemission spectroscopy study ${ }^{39}$ provided further compelling evidence for strong $e$-ph interaction in the cuprates. It revealed a fine phonon structure in the electron self-energy of underdoped $\mathrm{La}_{2-x} \mathrm{Sr}_{x} \mathrm{CuO}_{4}$ samples ${ }^{39,40}$ and a complicated isotope effect in the electron spectral function of $\mathrm{Bi} 2212$ that depended on the electron energy and momentum. ${ }^{41}$

With increasing phonon frequency the range of validity of the $1 / \lambda$ polaron expansion extends to smaller values of $\lambda .{ }^{11}$ As a result, the region of applicability of the MigdalEliashberg approach (even with vertex corrections) shrinks to smaller values of the coupling $\lambda<1$ with increasing $\omega$. Strong correlations between carriers might reduce this region further. ${ }^{25}$ Carriers in the fascinating novel materials are strongly coupled with high-frequency optical phonons, making small polarons and nonadiabatic effects relevant for hightemperature superconductivity and colossal magnetoresistance phenomena. Indeed the characteristic phonon energies 0.05-0.2 eV in cuprates, manganites, and in doped fullerenes are of the same order as the generally accepted values of the hopping integrals $t \simeq 0.1-0.3 \mathrm{eV}{ }^{42}$

The continued interest in polarons extends beyond physical description of low-mobility conductors such as the oxides or doped polymers. The field has been a testing ground for analytical and numerical techniques for several decades. In the past 25 years, several families of powerful numerical methods have been developed and successfully applied to one-, two-, and multiple-polaron lattice models. These are the quantum Monte Carlo (QMC) simulations, ${ }^{19,43-50}$ exact diagonalization of finite clusters, ${ }^{23,25,26,51}$ advanced variational methods, 4,30,31,34,35 and the density-matrix renormalization group. ${ }^{52}$ Many methods have been developed so far as to enable reliable calculation of not only static and thermodynamic polaron properties, but also of the effective mass, spectrum, and, in some cases, the spectral function of the polaron.

At the same time, the bulk of the lattice polaron studies have been limited to the short-range electron-phonon interactions described by the Holstein model. ${ }^{53}$ In numerical calculations, the locality of the interaction usually simplifies the algorithm and reduces the finite-size errors. However, as pointed out by two of us (A.S.A. and P.E.K.), ${ }^{36}$ the Holstein model is not a typical but an extreme polaron model because the screening length is normally larger than the lattice constant in doped insulators. It yields the highest possible value of the polaron mass in the strong coupling limit, if lattice vibrations are isotropic or polarised perpendicular to the hopping direction. ${ }^{54}$ With an on-site electron-phonon interaction, during every polaron hop the existing lattice deformation has to relax completely to the undeformed state, while a full deformation has to form again at the new location of the particle. Such a process results in the exponentially small overlap between the initial and the final states, and in an exponentially large effective mass with $\gamma=1$. Real ionic solids with low density of free carriers are characterized by poor screening and are more appropriately described by a longrange electron-phonon interaction. Thus the lattice Fröhlich model introduced in Ref. 36 is intermediate between the extremes of the Holstein and Fröhlich ${ }^{55}$ limits. On one hand, it is a lattice model (similar to the Holstein one), and the ratio of the hopping integral to the phonon frequency is an important parameter. On the other hand, the electron-phonon interaction is long range, as in the Fröhlich model. It was shown in Ref. 36 that in this intermediate case the polaron mass still grows exponentially with the polaron binding energy $E_{p}$ but the parameter $\gamma$ is now less than unity. That leads to much reduced numerical values of the polaron mass, hence the term "mobile small polaron." The model was further studied by numerical cluster diagonalization ${ }^{56}$ and $1 / \lambda$ expansion. ${ }^{11,57}$ In addition, the two-particle model with nonlocal electron-phonon interactions was studied variationally $^{58}$ and by the $1 / \lambda$ expansion technique. ${ }^{11,59}$ These studies confirmed the original conclusion that a longrange interaction significantly reduces the effective mass of the carrier, polaron or bipolaron, sometimes by several orders of magnitude, in comparison with the Holstein model. It also makes the self-trapping transition more gradual as a function of $\lambda$, and better describable by the Lang-Firsov theory. ${ }^{15}$ These findings are in agreement with some earlier studies on long-range interactions in narrow-band models. ${ }^{60}$

In this paper, we further generalize the lattice Fröhlich model of Ref. 36 to electron-phonon interaction of some finite radius $R$. We perform a systematic study of the single polaron problem in one dimension as a function of $R$. In the local limit $R \rightarrow 0$ we recover the results of the Holstein model obtained in the past by various methods mentioned above. In the infinite- $R$ limit the original AK model and its results are fully recovered as well. Our computational tool will be the continuous-time path-integral quantum Monte Carlo algorithm developed previously by one of us. ${ }^{48}$ This method is particularly suited for investigating long-range electron-phonon interactions because the phonon degrees of freedom are integrated out analytically. Thus the shape of the interaction does not complicate the algorithm at all, but simply modifies the weight function of a Monte Carlo configuration. The method works on infinite lattices and in arbitrary dimensions, eliminating finite-size errors; there is also no truncation of the phonon Fock space. The method is also free from finite-time-step errors because it is formulated in continuous time. The method enables unbiased calculation (i.e., no numerical errors besides statistical fluctuations) of the polaron energies, effective mass, spectrum, density of states, isotope exponents, the number of excited phonons, and other quantities.

In addition to presenting results on the finite-radius Fröhlich model, we use the present paper to explain many technical details of the polaron QMC method, ${ }^{48}$ which have not previously been published. The electron-phonon Hamiltonian is introduced in Sec. II. In Sec. III we describe the continuous-time Monte Carlo method. In Secs. IV-VI we present the numerical results for the energy, effective mass, 
number of dressing phonons, and isotope exponents of lattice polarons for different $R$, and compare them with weakcoupling and strong-coupling analytical results and with numerical results of other authors. Section VII summarizes our conclusions.

\section{ELECTRON-PHONON MODEL}

\section{A. General model Hamiltonian}

The electron-phonon model under investigation represents a single electron interacting with all the ions of an infinite hypercubic lattice, with one vibrational degree of freedom per unit cell. The Hamiltonian takes the form

$$
H=H_{e}+H_{\mathrm{ph}}+H_{e-\mathrm{ph}},
$$

where

$$
\begin{gathered}
H_{e}=-t \sum_{\left\langle\mathbf{n n}^{\prime}\right\rangle} c_{\mathbf{n}^{\dagger}}^{\dagger} c_{\mathbf{n}^{\prime}}, \\
H_{\mathrm{ph}}=\frac{1}{2 M} \sum_{\mathbf{m}} P_{\mathbf{m}}^{2}+\frac{M \omega^{2}}{2} \sum_{\mathbf{m}} \xi_{\mathbf{m}}^{2},
\end{gathered}
$$

and

$$
H_{e-\mathrm{ph}}=-\sum_{\mathbf{n m}} f_{\mathbf{m}}(\mathbf{n}) c_{\mathbf{n}}^{\dagger} c_{\mathbf{n}} \xi_{\mathbf{m}} .
$$

The free-electron term $H_{e}$ describes the movement of a single electron through the lattice by the process of nearestneighbor hopping. Here the operator $c_{\mathbf{n}}^{\dagger}$ creates an electron on site $\mathbf{n}$, the operator $c_{\mathbf{n}^{\prime}}$ destroys an electron on site $\mathbf{n}^{\prime}$, and $\left\langle\mathbf{n n}^{\prime}\right\rangle$ denotes pairs of nearest-neighbor sites. The phonon term $H_{\mathrm{ph}}$ represents the vibrations of the lattice ions. Here the operator $\xi_{\mathrm{m}}$ is the displacement of the $\mathbf{m}$ th ion from its equilibrium position, and $P_{\mathbf{m}}=-i \hbar \partial / \partial \xi_{\mathbf{m}}$ its momentum. It is assumed that the ions, each of ionic mass $M$, are noninteracting and so have the same characteristic (phonon) frequency $\omega$. The final part of the Hamiltonian, the electronphonon term $H_{e-\mathrm{ph}}$, is of the "density-displacement" type, where the interaction energy between the electron and the $\mathbf{m}$ th ion is proportional to $\xi_{\mathbf{m}}$ (the displacement of the $\mathbf{m}$ th ion from its equilibrium position). Here $c_{\mathbf{n}}^{\dagger} c_{\mathbf{n}}$ is the electron number operator, and $f_{\mathbf{m}}(\mathbf{n})$ is interpreted as the interaction force between the electron on site $\mathbf{n}$ and the $\mathbf{m}$ th lattice ion.

The model is parametrized by two dimensionless quantities. The first is the dimensionless phonon frequency

$$
\bar{\omega}=\hbar \omega / t .
$$

The second is related to the small-polaron binding energy $E_{p}$, derived in Sec. VI, which serves as a natural and convenient measure of the strength of the electron-phonon interaction. The dimensionless electron-phonon coupling constant is defined as

$$
\lambda=\frac{E_{p}}{z t}=\frac{1}{2 M \omega^{2} z t} \sum_{\mathbf{m}} f_{\mathbf{m}}^{2}(0),
$$

where $z t$ is the bare-electron half bandwidth, with $z$ the lattice coordination number.

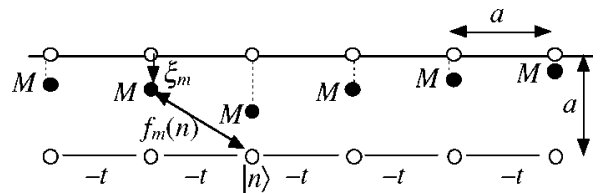

FIG. 1. Geometry of the Fröhlich model (1)-(4) shown in one dimension. The mobile charge carrier moves on the lower chain with nearest-neighbor hopping integral $t$ and interacts with all the ions of the upper chain. The displacements $\xi_{m}$ of the ions are polarized in a direction perpendicular to the chains.

\section{B. Discrete Fröhlich model}

Some time ago Alexandrov and Kornilovitch proposed a long-range discrete Fröhlich interaction ${ }^{36}$ to describe the interaction between a hole and the apical oxygen ions in high$T_{\mathrm{c}}$ superconducting materials. The model is depicted in Fig. 1 for the one-dimensional case. The mobile carrier (electron or hole) may hop from site to nearest-neighbor site along the lower chain. The chain consists of an infinite number of lattice sites with lattice constant $a$. The electron interacts with all the ions which reside at the lattice sites of a similar chain that is parallel to the first. The separation of the two chains is equal to the lattice constant $a$. We assume that the vibrations of the ions are polarized in a direction that is perpendicular to the chains, and that the ions do not interact with each other.

Let us find the appropriate form for the interaction force $f_{m}(n)$ between the mobile charge-carrier on the $n$th site (of the lower chain) and the $m$ th ion (of the upper chain). Since both $m$ and $n$ are measured in units of $a$, we choose from this point on to take $a=1$. The presence of the charge-carrier displaces the $m$ th ion by a small distance $\xi_{m}$ in a direction perpendicular to the chain, as shown in Fig. 1. By expanding the Coulomb potential in powers of $\xi_{m}$, we deduce ${ }^{36}$ that the Hamiltonian for the discrete Fröhlich model is that of our generalized model Hamiltonian (1) with the electron-phonon interaction force having the form

$$
f_{m}(n)=\frac{\kappa}{\left[(m-n)^{2}+1\right]^{3 / 2}}
$$

with a constant $\kappa$. Physically, this model was proposed in order to represent the interaction between a hole in the copper-oxygen layer (lower chain) and the apical oxygens in the ionic layer (upper chain) contained within the structure of certain doped high- $T_{\mathrm{c}}$ superconductors such as $\mathrm{YBa}_{2} \mathrm{Cu}_{3} \mathrm{O}_{6+x} \cdot{ }^{36}$ These materials are highly anisotropic due to the fact that the holes are sharply localized in the copperoxygen layer, giving rise to poor conduction in the $c$ direction (normal to copper-oxygen layer). This leads to very poor screening of the electron-phonon interaction in the $c$ direction, and almost complete screening in the $a-b$ plane. This justifies the restriction to phonon modes polarized in the $c$ direction.

\section{Screened Fröhlich model}

Our aim in this paper is to investigate the way in which the shape of the long-range electron-phonon interaction af- 


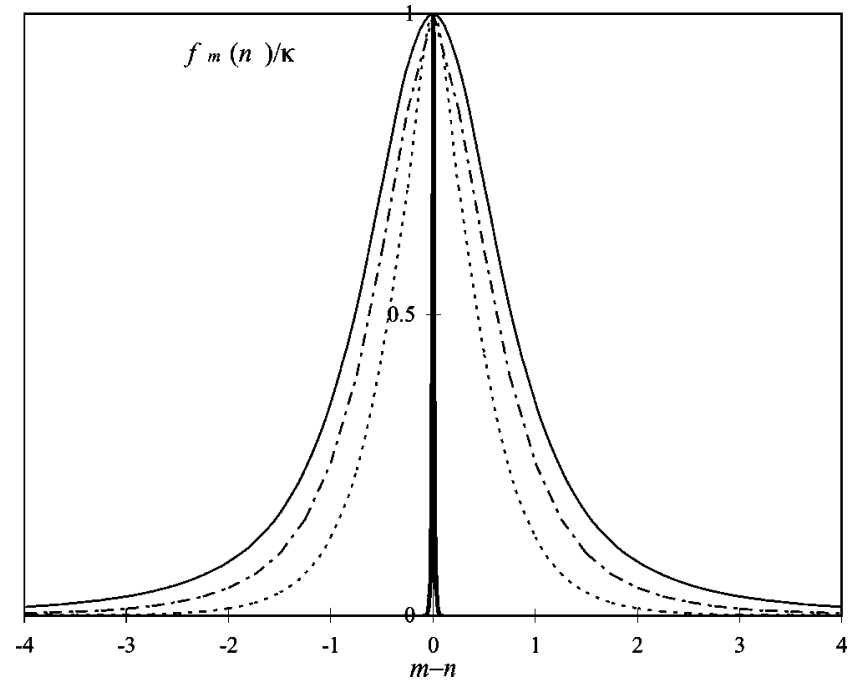

FIG. 2. The shape of the screened Fröhlich interaction force, (8), at screening lengths of $R_{\mathrm{sc}} \rightarrow 0$ (bold line, Holstein interaction), $R_{\mathrm{sc}}=1$ (dashed line), $R_{\mathrm{sc}}=3$ (dot-dashed line), and $R_{\mathrm{sc}} \rightarrow \infty$ (thin line, nonscreened Fröhlich interaction).

fects the properties of the polaron. It is therefore interesting to study the screened Fröhlich model, in which the screening effect due to the presence of other electrons in the lattice is taken into account from within $f_{m}(n)$. Accordingly, let us define the interaction force for the screened Fröhlich model as

$$
f_{m}(n)=\frac{\kappa}{\left[(m-n)^{2}+1\right]^{3 / 2}} \exp \left(-\frac{|m-n|}{R_{\mathrm{sc}}}\right),
$$

where $R_{\mathrm{sc}}$ is the screening length. That is, the screened force is the unscreened force multiplied by an exponential damping factor. Increasing the value of $R_{\mathrm{sc}}$ decreases the screening effect and thus increases the width of the interaction force.

The Holstein model describes an electron that interacts only with the oscillator it currently occupies ("short-range" interaction). This may be regarded as a special case of Eq. (8) with $R_{\mathrm{sc}} \rightarrow 0$, so that $f_{m}(n)=-\kappa \delta_{m n}$. Simply by altering the value of the parameter $R_{\mathrm{sc}}$, we can easily cross over from the Holstein model, through the screened Fröhlich model, to the unscreened Fröhlich model, in a universal manner. In this paper we consider the following four cases in one dimension: (1) Holstein model with $R_{\mathrm{sc}} \rightarrow 0$, (2) screened Fröhlich model, with $R_{\mathrm{sc}}=1$, (3) screened Fröhlich model, with $R_{\mathrm{sc}}$ $=3$, and (4) Unscreened Fröhlich model with $R_{\mathrm{sc}} \rightarrow \infty$.

The shapes of the electron-phonon interaction force $f_{m}(n)$ for each of the above screening lengths are shown in Fig. 2. Note that, based on calculations involving the dynamic properties of the polaron response, ${ }^{11}$ the amount of screening we impose here is greater than that expected in the high- $T_{\mathrm{c}}$ compounds.

\section{PATH INTEGRAL APPROACH}

\section{A. Effective mass using a partial partition function}

The effective mass of the polaron $m^{*}$ is defined for the isotropic or one-dimensional case as ${ }^{48}$

$$
\left(m^{*}\right)^{-1}=\lim _{P \rightarrow 0} \frac{\partial^{2} E(P)}{\partial P^{2}},
$$

where $E(P)$ is the ground-state energy for total momentum $P$ (sum of the momenta of the electron and all the phonons).

The evaluation of $\left(m^{*}\right)^{-1}$ by differentiating QMC energies is not practical within our approach because a minus-sign problem arises for finite momentum, exacerbating the errors already present in such a procedure. The usual means of extracting dynamical properties (such as the effective mass) from QMC simulation is by making use of some kind of analytical continuation from imaginary to real time. However, it is possible to infer $m^{*}$ directly from QMC simulation by considering electron trajectories with twisted (rather than periodic) boundary conditions in imaginary time. Kornilovitch ${ }^{48}$ showed (for the isotropic or one-dimensional case) that

$$
\frac{m_{0}}{m^{*}}=\lim _{\beta \rightarrow \infty} \frac{1}{2 \beta t a^{2}} \frac{\sum_{\Delta \mathbf{r}}(\Delta \mathbf{r})^{2} Z_{\Delta \mathbf{r}}}{\sum_{\Delta \mathbf{r}} Z_{\Delta \mathbf{r}}},
$$

where $m_{0}=\hbar^{2} /\left(2 t a^{2}\right)$ is the bare electron mass and

$$
Z_{\Delta \mathbf{r}}=\int d^{N} \boldsymbol{\xi}\left\langle\left\{\xi_{\mathbf{m}+\mathbf{r}^{\prime}-\mathbf{r}}\right\}, \mathbf{r}^{\prime}\left|e^{-\beta H}\right|\left\{\xi_{\mathbf{m}}\right\}, \mathbf{r}\right\rangle,
$$

is a "partial partition function" (which is similar in form to the total partition function of the system), with

$$
\int d^{N} \boldsymbol{\xi}=\prod_{\mathbf{m}=1}^{N}\left[\int_{-\infty}^{\infty} d \xi_{\mathbf{m}}\right] .
$$

Here $|\mathbf{r}\rangle$ is the electron basis, $\left|\left\{\xi_{\mathbf{m}}\right\}\right\rangle=\left|\xi_{1}, \xi_{2}, \xi_{3}, \ldots, \xi_{N}\right\rangle$ is the ionic displacement basis, and the summations over $\Delta \mathbf{r}$ includes all possible values of $\Delta \mathbf{r}=\mathbf{r}^{\prime}-\mathbf{r}$.

Given Eq. (10), the effective mass may be obtained from QMC simulation by taking the statistical average of $(\Delta \mathbf{r})^{2}$, sampled over trajectories of the path integral formed from $Z_{\Delta \mathrm{r}}$. The dissimilarity between the "bra" and "ket" states in Eq. (11) produces a path integral of $Z_{\Delta \mathbf{r}}$ having twisted (rather than the usual periodic) boundary conditions. Note that, since we need only consider the case of $\mathbf{P}=0$, there is no sign problem.

\section{B. Continuous imaginary time}

QMC schemes have recently been developed that are implemented directly in continuous imaginary time for lattice models, ${ }^{19,50,61}$ eliminating the problematic finite-timestep error associated with the traditional discrete-time approach. The partial partition function $Z_{\Delta \mathbf{r}}$ in Eq. (11) is given in continuous-imaginary-time path-integral form as ${ }^{48}$

$$
Z_{\Delta \mathbf{r}}=\int_{\mathrm{tw}} \mathcal{D} \boldsymbol{\xi} \mathcal{D} \mathbf{r} \exp (S)
$$

where the phonon action reads 


$$
\begin{aligned}
S= & \sum_{\mathbf{m}} S_{\mathbf{m}}=\sum_{\mathbf{m}} \int_{0}^{\beta} d \tau\left[-\frac{M}{2 \hbar^{2}} \dot{\xi}_{\mathbf{m}}^{2}(\tau)-\frac{M \omega^{2}}{2} \xi_{\mathbf{m}}^{2}(\tau)\right. \\
& \left.+f_{\mathbf{m}}[\mathbf{r}(\tau)] \xi_{\mathbf{m}}(\tau)\right],
\end{aligned}
$$

with $\dot{\xi}_{\mathbf{m}}(\tau)=\partial \xi_{\mathbf{m}}(\tau) / \partial \tau$. In forming the path integral above, an imaginary-time dimension $\tau$ has been introduced, having the range $0 \leqslant \tau \leqslant \beta$. The electron and phonon coordinates are represented as continuous functions of imaginary time $\mathbf{r}(\tau)$ and $\xi_{\mathrm{m}}(\tau)$, which can be interpreted as continuous trajectories in $\tau$.

The symbol $\int_{\text {tw }}$ in Eq. (13) represents the integration over all possible trajectories under twisted boundary conditions in imaginary time. The "end states" of the individual trajectories, which are identified with the states $\left\langle\left\{\xi_{\mathbf{m}+\mathbf{r}^{\prime}-\mathbf{r}}\right\}, \mathbf{r}^{\prime}\right|$ and $\left|\left\{\xi_{\mathbf{m}}\right\}, \mathbf{r}\right\rangle$ in Eq. (11), are given by ${ }^{48}$

$$
\begin{gathered}
\left|\left\{\xi_{\mathbf{m}}(0)\right\}, \mathbf{r}(0)\right\rangle=\left|\left\{\xi_{\mathbf{m}}\right\}, \mathbf{r}\right\rangle \\
\left|\left\{\xi_{\mathbf{m}}(\beta)\right\}, \mathbf{r}(\beta)\right\rangle=\left|\left\{\xi_{\mathbf{m}+\Delta \mathbf{r}}\right\}, \mathbf{r}+\Delta \mathbf{r}\right\rangle,
\end{gathered}
$$

that is, the final state $(\tau=\beta)$ is the initial state $(\tau=0)$ with all the coordinates (electron and all phonons) shifted by $\Delta \mathbf{r}$.

We may decompose the trajectory $\xi_{\mathbf{m}}(\tau)$ in Eq. (14) into the sum of a the classical path (the trajectory that extremizes $S_{\mathbf{m}}$ ) and a deviation (or "quantum fluctuation") from it. The part of $S_{\mathbf{m}}$ that contains no terms involving quantum fluctuation is the classical action. The classical action is an important quantity, and is given by ${ }^{48,62}$

$$
\begin{aligned}
S_{\mathbf{m}}^{\mathrm{cl}}= & \frac{M \omega}{2 \hbar \sinh (\hbar \omega \beta)}\left\{-\left[\xi_{\mathbf{m}}^{2}(0)+\xi_{\mathbf{m}}^{2}(\beta)\right] \cosh (\hbar \omega \beta)\right. \\
& \left.+2 \xi_{\mathbf{m}}(0) \xi_{\mathbf{m}}(\beta)\right\}+\xi_{\mathbf{m}}(0) B_{\mathbf{m}}(\tau)+\xi_{\mathbf{m}}(\beta) C_{\mathbf{m}}(\tau) \\
& +\frac{\hbar^{2}}{2 M} \int_{0}^{\beta} \int_{0}^{\beta} d \tau d \tau^{\prime} f_{\mathbf{m}}[\mathbf{r}(\tau)] G\left(\tau, \tau^{\prime}\right) f_{\mathbf{m}}\left[\mathbf{r}\left(\tau^{\prime}\right)\right],
\end{aligned}
$$

where

$$
\begin{gathered}
B_{\mathbf{m}}(\tau) \equiv \int_{0}^{\beta} d \tau \frac{\sinh [\hbar \omega(\beta-\tau)]}{\sinh (\hbar \omega \beta)} f_{\mathbf{m}}[\mathbf{r}(\tau)], \\
C_{\mathbf{m}}(\tau) \equiv \int_{0}^{\beta} d \tau \frac{\sinh (\hbar \omega \tau)}{\sinh (\hbar \omega \beta)} f_{\mathbf{m}}[\mathbf{r}(\tau)]
\end{gathered}
$$

and the Green function is

$$
\begin{aligned}
G\left(\tau, \tau^{\prime}\right)= & \frac{1}{\hbar \omega \sinh (\hbar \omega \beta)} \\
& \times \begin{cases}\sinh (\hbar \omega \tau) \sinh \left[\hbar \omega\left(\beta-\tau^{\prime}\right)\right], & 0<\tau<\tau^{\prime}, \\
\sinh [\hbar \omega(\beta-\tau)] \sinh \left(\hbar \omega \tau^{\prime}\right), & \tau^{\prime}<\tau<\beta .\end{cases}
\end{aligned}
$$

Note that the phonon coordinates in Eq. (16) are those of the end-points only: $\xi_{\mathbf{m}}(0)$ and $\xi_{\mathbf{m}}(\beta)$.

\section{Analytical phonon integration}

We wish to integrate out the phonon degrees of freedom from the problem analytically, that is, perform the phonon path integral

$$
I_{\mathrm{tw}}=\int_{\mathrm{tw}} \mathcal{D} \boldsymbol{\xi} \exp \left(\sum_{\mathbf{m}} S_{\mathbf{m}}\right)=c_{\mathrm{tw}} \int_{\mathrm{tw}} d \boldsymbol{\xi} \exp \left(\sum_{\mathbf{m}} S_{\mathbf{m}}^{\mathrm{cl}}\right),
$$

where the nonclassical part of $S$ (terms involving quantum fluctuation) integrates to an unimportant constant ${ }^{48} c_{\mathrm{tw}}$, reducing the problem to the integration of the classical action $S_{\mathrm{m}}^{\mathrm{cl}}$. The integration must be performed under twisted boundary conditions in imaginary time. Accordingly, we impose the constraints

$$
\xi_{\mathbf{m}}(0)=\xi_{\mathbf{m}}, \quad \xi_{\mathbf{m}}(\beta)=\xi_{\mathbf{m}-\Delta \mathbf{r}}
$$

on $S_{\mathbf{m}}^{\mathrm{cl}}$ in Eq. (16), which one can see produces mixed variable terms involving $\xi_{\mathbf{m}} \xi_{\mathbf{m}-\Delta \mathrm{r}}$. The phonon integration cannot directly be performed in this form. However, we may proceed by transforming $\xi_{\mathbf{m}}$ into real Fourier components $a_{\mathbf{q}}$ and $b_{\mathbf{q}}$ :

$$
\xi_{\mathbf{m}}=\frac{1}{\sqrt{D N}} \sum_{\mathbf{q}}\left(a_{\mathbf{q}}+i b_{\mathbf{q}}\right) e^{i \mathbf{q} \cdot \mathbf{m}},
$$

where $D$ is the dimensionality of the lattice and $D N$ is the total number of phonon degrees of freedom. In this representation, the transformed action $S_{\mathbf{q}}^{\mathrm{cl}}$ is diagonal, and so the phonon path integral in Eq. (20) decomposes to the product of single variable integrals according to

$$
I_{\mathrm{tw}}=c_{\mathrm{tw}} \prod_{\mathbf{q}} \int_{\mathrm{tw}} d a_{\mathbf{q}} d b_{\mathbf{q}} \exp \left(S_{\mathbf{q}}^{\mathrm{cl}}\right) .
$$

After performing the Gaussian integration (in $a_{\mathbf{q}}$ and $b_{\mathbf{q}}$ ) the result is

$$
I_{\mathrm{tw}}=c_{\mathrm{tw}}\left[\frac{\pi \hbar \sinh (\hbar \omega \beta)}{M \omega[\cosh (\hbar \omega \beta)-\cos (\mathbf{q} \cdot \Delta \mathbf{r})]}\right]^{D N / 2} \exp A,
$$

where

$$
\begin{aligned}
A= & \frac{\hbar \sinh (\hbar \omega \beta) \sum_{\mathbf{m}} B_{\mathbf{m}}\left(C_{\mathbf{m}-\Delta \mathbf{r}}-C_{\mathbf{m}}\right)}{2 M \omega[\cosh (\hbar \omega \beta)-\cos (\mathbf{q} \cdot \Delta \mathbf{r})]} \\
& +\frac{\hbar \sinh (\hbar \omega \beta) \sum_{\mathbf{m}}\left(B_{\mathbf{m}}+C_{\mathbf{m}}\right)^{2}}{4 M \omega[\cosh (\hbar \omega \beta)-\cos (\mathbf{q} \cdot \Delta \mathbf{r})]} \\
& +\sum_{\mathbf{m}} \frac{\hbar^{2}}{2 M} \int_{0}^{\beta} \int_{0}^{\beta} d \tau d \tau^{\prime} f_{\mathbf{m}}[\mathbf{r}(\tau)] G\left(\tau, \tau^{\prime}\right) f\left[\mathbf{r}\left(\tau^{\prime}\right)\right],
\end{aligned}
$$

which does not contain any phonon degrees of freedom. We have thus transformed the problem from that of an electron interacting with many phonons to that of an electron with retarded self-interaction, which allows the QMC method to be applied effectively. 


\section{Low-temperature limit}

The result above may be conveniently rendered into the required low-temperature limit using $\cosh \hbar \omega \beta \approx \sinh \hbar \omega \beta$ $\approx \frac{1}{2} e^{\hbar \omega \beta} \gg 1$, to give

$$
I_{\mathrm{tw}} \propto \exp A=\exp \left(A_{\mathrm{per}}+\Delta A\right)
$$

where

$$
\begin{aligned}
A_{\mathrm{per}}= & \frac{z \lambda \bar{\omega}}{2 \Phi_{0}(0,0)} \int_{0}^{\bar{\beta}} \int_{0}^{\bar{\beta}} d \bar{\tau} d \bar{\tau}^{\prime} e^{-\bar{\omega}(\bar{\beta} / 2)}\left(e^{\bar{\omega}\left(\bar{\beta} / 2-\left|\bar{\tau}-\bar{\tau}^{\prime}\right|\right)}\right. \\
& \left.+e^{-\bar{\omega}\left(\bar{\beta} / 2-\left|\bar{\tau}-\bar{\tau}^{\prime}\right|\right)}\right) \Phi_{0}\left[\mathbf{r}(\bar{\tau}), \mathbf{r}\left(\bar{\tau}^{\prime}\right)\right]
\end{aligned}
$$

is the low-temperature action for periodic boundary conditions and

$$
\begin{aligned}
\Delta A= & \frac{z \lambda \bar{\omega}}{\Phi_{0}(0,0)} \int_{0}^{\bar{\beta}} \int_{0}^{\bar{\beta}} d \bar{\tau} d \bar{\tau}^{\prime} e^{-\bar{\omega} \bar{\tau}} e^{-\bar{\omega}\left(\bar{\beta}-\bar{\tau}^{\prime}\right)}\left\{\Phi_{\Delta \mathbf{r}}\left[\mathbf{r}(\bar{\tau}), \mathbf{r}\left(\bar{\tau}^{\prime}\right)\right]\right. \\
& \left.-\Phi_{0}\left[\mathbf{r}(\bar{\tau}), \mathbf{r}\left(\bar{\tau}^{\prime}\right)\right]\right\}
\end{aligned}
$$

is the correction for twisted boundary conditions, in dimensionless form. Here $\bar{\tau}=t \tau$ and $\bar{\beta}=t \beta$ define dimensionless imaginary time; the parameters $\bar{\omega}$ and $\lambda$ are defined in Eqs. (5) and (6), respectively; and the lattice summation is defined as

$$
\Phi_{\Delta \mathbf{r}}\left[\mathbf{r}(\bar{\tau}), \mathbf{r}\left(\bar{\tau}^{\prime}\right)\right]=\sum_{\mathbf{m}} \bar{f}_{\mathbf{m}}[\mathbf{r}(\bar{\tau})] \bar{f}_{\mathbf{m}+\Delta \mathbf{r}}\left[\mathbf{r}\left(\bar{\tau}^{\prime}\right)\right]
$$

Note that the dimensionless quantity $\bar{f}_{\mathbf{m}}(\mathbf{n})$ represents the shape or form of the electron-phonon interaction force, defined via the decomposition

$$
f_{\mathbf{m}}(\mathbf{n})=\kappa \bar{f}_{\mathbf{m}}(\mathbf{n}),
$$

where $\kappa=\left\{2 z \lambda M t^{3} \bar{\omega}^{2} /\left[\hbar^{2} \Sigma_{\mathbf{m}} \bar{f}_{\mathbf{m}}^{2}(0)\right]\right\}^{1 / 2}$ takes the dimensions of force.

\section{CONTINUOUS-TIME MONTE CARLO}

\section{A. Algorithm}

Traditionally, path-integral QMC simulation is implemented in discrete imaginary time, where the trajectory is represented by the position of the electron in each of a large number of imaginary-time slices. The use of discrete time introduces the problematic finite-time-step systematic error, which scales with the square of the time-slice width.

A path-integral QMC scheme implemented directly in continuous imaginary time has been developed for systems with a discrete basis. ${ }^{19,61}$ Here, the electron trajectory is represented as finite intervals of imaginary time in which the system remains in a particular state, separated by sporadic transitions from one state to another (an electron hop). The points in imaginary time at which the state of the system changes are called "kinks," as shown in Fig. 3. It is necessary to consider the statistics governing different directions of kink independently of one another. For our one-dimensional case with nearest-neighbor hopping, we need only consider single left and right kinks. The use of continuous time com-

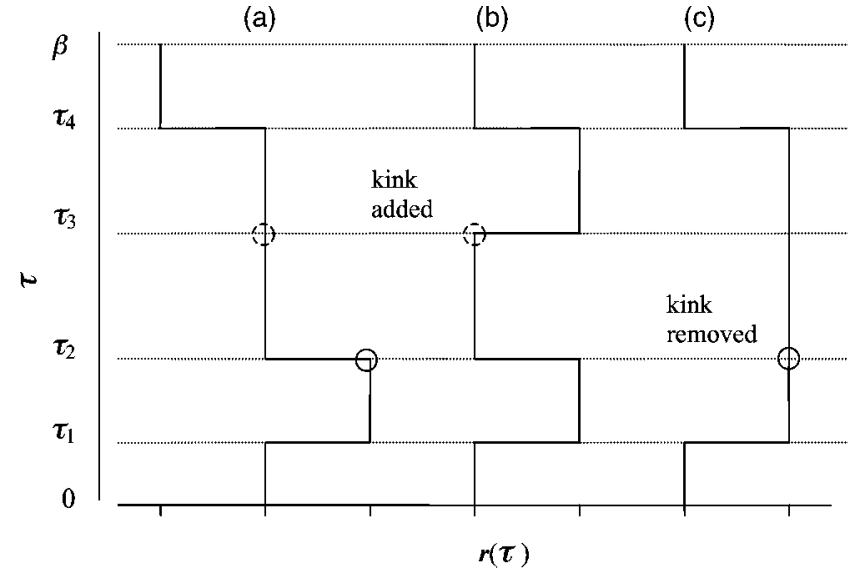

FIG. 3. Illustration of a one-dimensional electron trajectory in imaginary time. The point in imaginary time at which the electron hops to a neighboring site is known as a kink. (a) Shows a trajectory with three kinks: occurring at $\tau_{1}, \tau_{2}$, and $\tau_{4}$. (b) The same trajectory, but with a kink added at time $\tau_{3}$. The entire trajectory above $\tau_{3}$ is shifted by one lattice parameter. (c) The same trajectory as in (a) but with the kink at $\tau_{2}$ removed. Again, the entire trajectory above $\tau_{2}$ is shifted accordingly.

pletely eliminates the finite-time-step error, rendering the scheme "numerically exact."

If $N_{s}$ is the number of kinks of direction $s$, we wish to generate random states according to the Monte Carlo weight

$$
w\left(\left\{N_{s}\right\}\right)=w_{\mathrm{el}}\left(\left\{N_{s}\right\}\right) w_{\mathrm{ph}}\left(\left\{N_{s}\right\}\right),
$$

where the weight from the electron subsystem

$$
w_{\mathrm{el}}\left(\left\{N_{s}\right\}\right)=\prod_{s} \frac{(t \beta)^{N_{s}} e^{-t \beta}}{N_{s} !}
$$

is given by the Poisson distribution, and the phonon-induced weight

$$
w_{\mathrm{ph}}\left(N_{s}\right)=\exp \left[A\left(N_{s}\right)\right]
$$

is given by Eq. (26).

Proposed changes to the shape of the trajectory are generated by the addition or deletion of single kinks. This is sufficient in practice. In order to increase efficiency one might also consider changing a kink-direction, repositioning a kink in imaginary time, or altering the temporal ordering of the kinks. The Metropolis method ${ }^{63,64}$ accepts or rejects the trial change from state $\mu$ to state $\mu^{\prime}$ with a transition probability $P\left(\mu \longrightarrow \mu^{\prime}\right)=g\left(\mu \rightarrow \mu^{\prime}\right) a\left(\mu \longrightarrow \mu^{\prime}\right)$, where $g\left(\mu \rightarrow \mu^{\prime}\right)$ is the sampling distribution and $a\left(\mu \rightarrow \mu^{\prime}\right)$ is the acceptance probability. For the case of $N_{s} \geqslant 1$ (one or more kinks exist), we choose $g\left(N_{s}+1 \rightarrow N_{s}\right)=g\left(N_{s} \rightarrow N_{s}+1\right)=1 / 2$, and so the acceptance probability is given by

$$
a_{\text {add }}\left(N_{s} \rightarrow N_{s}+1\right)=\min \left\{1, \frac{g\left(N_{s}+1 \rightarrow N_{s}\right)}{g\left(N_{s} \rightarrow N_{s}+1\right)} \frac{W\left(N_{s}+1\right)}{W\left(N_{s}\right)}\right\}
$$




$$
=\min \left\{1, \frac{t \beta}{N_{s}+1} \exp \left[A\left(N_{s}+1\right)-A\left(N_{s}\right)\right]\right\}
$$

to add a kink, and similarly

$$
a_{\mathrm{rem}}\left(N_{s}+1 \rightarrow N_{s}\right)=\min \left\{1, \frac{N_{s}+1}{t \beta} \exp \left[A\left(N_{s}\right)-A\left(N_{s}+1\right)\right]\right\}
$$

to remove a kink of direction $s$. For the case of $N_{s}=0$, we can only add a kink, and so $g(0 \rightarrow 1)=1$, which gives

$$
a_{\text {add }}(0 \rightarrow 1)=\min \left\{1, \frac{t \beta}{2} \exp [A(1)-A(0)]\right\}
$$

and

$$
a_{\mathrm{rem}}(1 \rightarrow 0)=\min \left\{1, \frac{2}{t \beta} \exp [A(0)-A(1)]\right\} .
$$

The continuous-imaginary-time QMC step used in this work has the following structure.

(1) Randomly select a kink direction $s$ for the trial change. In the case of a one-dimensional system, this is left or right.

(2) Propose a change to the trajectory by randomly selecting whether to add a new kink (at a random imaginary time) or to remove an existing kink (selected in a random fashion). This is done according to the selection probabilities $g\left(N_{s}\right.$ $\left.+1 \rightarrow N_{s}\right)$ and $g\left(N_{s} \rightarrow N_{s}+1\right)$.

(3) Accept or reject the proposed change with probability $a_{\text {add }}\left(N_{s} \rightarrow N_{s}+1\right)$ if adding, or $a_{\text {rem }}\left(N_{s}+1 \rightarrow N_{s}\right)$ if removing a kink.

(4) If the change has been accepted, then the entire trajectory that lies "above" the kink (i.e., from the imaginary time of the kink to $\beta$ ) is shifted across accordingly. If the proposed change has been rejected, then the trajectory is left untouched.

\section{B. Analytical integration over kinks}

The Metropolis algorithm requires the action, which involves a double integration in imaginary time, to be computed on each Monte Carlo step. The fact that the trajectory consists of a series of single kinks, between which the trajectory is a straight line $[\mathbf{r}(\bar{\tau})$ is constant $]$, leads us to decompose the action $A$ in Eq. (26) into segments according to

$$
A=\sum_{j=1}^{N_{s}+1} A_{j j}+2 \sum_{j=1}^{N_{s}} \sum_{k=j+1}^{N_{s}+1} A_{j k}
$$

where $j$ and $k$ label the kinks (along trajectories corresponding with $\tau$ and $\tau^{\prime}$, respectively, in the double integration), such that $\bar{\tau}_{j}$ is the imaginary time at which the $j$ th kink occurs, with $\bar{\tau}_{0}=0$ and $\bar{\tau}_{N_{\mathrm{s}}+1}=\bar{\beta}$. We treat the diagonal $A_{j j}$ and off-diagonal segments $A_{j k}$ separately in order to increase efficiency. Each $A_{j k}$ involves the range of imaginary time between successive kinks of $\bar{\tau}_{j-1} \leqslant \bar{\tau} \leqslant \bar{\tau}_{j}$ and $\bar{\tau}_{k-1} \leqslant \bar{\tau}^{\prime} \leqslant \bar{\tau}_{k}$, in which the electron coordinate is fixed at $\mathbf{r}(\bar{\tau})=\mathbf{r}\left(\bar{\tau}_{j-1}\right)$ and $\mathbf{r}\left(\bar{\tau}^{\prime}\right)=\mathbf{r}\left(\bar{\tau}_{k-1}\right)$, respectively. Thus the value of the lattice summation $\Phi_{\Delta \mathbf{r}}\left[\mathbf{r}\left(\bar{\tau}_{j-1}\right), \mathbf{r}\left(\bar{\tau}_{k-1}\right)\right]$ given by Eq. (29), has a constant value, allowing the double integration appearing in Eqs. (27) and (28) to be treated analytically for each segment $A_{j k}$. The result after rearrangement reads

$$
\begin{aligned}
A= & \frac{\lambda z}{\Phi_{0}^{(0,0)}}\left\{\sum_{j=1}^{N_{s}+1}\left[A_{0}^{(j)} \Phi_{0}^{(j, j)}+A_{1}^{(j)} \Phi_{\Delta \mathbf{r}}^{(j, j)}\right]\right. \\
& \left.+\sum_{j=1}^{N_{s}+1} \sum_{k=j+1}^{N_{s}+1}\left[A_{2} \Phi_{0}^{(j, k)}+A_{3} \Phi_{\Delta \mathbf{r}}^{(j, k)}\right]\right\},
\end{aligned}
$$

where we have used the shorthand $\Phi_{\Delta \mathbf{r}}^{(j, k)}$ $=\Phi_{\Delta \mathbf{r}}\left[\mathbf{r}\left(\bar{\tau}_{j-1}\right), \mathbf{r}\left(\bar{\tau}_{k-1}\right)\right]$ for the lattice summation defined in Eq. (29), and

$$
\begin{gathered}
A_{0}^{(j)}=\frac{1}{\bar{\omega}}\left[\bar{\omega}\left(\bar{\tau}_{j}-\bar{\tau}_{j-1}\right)-K^{(j)}\right], \\
A_{1}^{(j)}=\frac{1}{\bar{\omega}} e^{-\bar{\omega}\left(\bar{\beta}+\bar{\tau}_{j-1}-\bar{\tau}_{j}\right)}, \\
A_{2}=\frac{1}{\bar{\omega}} K^{(j)} K^{(k)} e^{-\bar{\omega}\left(\bar{\tau}_{k-1}-\bar{\tau}_{j}\right)},
\end{gathered}
$$

and

$$
A_{3}=\frac{1}{\bar{\omega}} K^{(j)} K^{(k)} e^{-\bar{\omega}\left(\bar{\beta}-\bar{\tau}_{k}+\bar{\tau}_{j-1}\right)},
$$

where we have defined

$$
K^{(j)}=1-e^{-\bar{\omega}\left(\bar{\tau}_{j}-\bar{\tau}_{j-1}\right)}
$$

The action can thus be computed efficiently using this double summation over kinks.

For the models studied in this paper, $f_{\mathbf{m}}(\mathbf{n})$ depends only on the relative lattice distance $|\mathbf{m}-\mathbf{n}|$, and tends to zero (or is zero) at large distance. Consequently, the lattice summation, Eq. (29), is a function of the single variable $\mathbf{r}^{\prime}=\mathbf{r}_{2}-\mathbf{r}_{1}-\Delta \mathbf{r}$ only, namely,

$$
\Phi\left(\mathbf{r}^{\prime}\right)=\sum_{\mathbf{m}^{\prime}} \bar{f}\left(\mathbf{m}^{\prime}\right) \bar{f}\left(\mathbf{m}^{\prime}-\mathbf{r}^{\prime}\right)
$$

which can be evaluated for all possible values of $\mathbf{r}^{\prime}$ in advance of the simulation proper, improving the efficiency of the QMC scheme.

\section{Physical observables}

We consider four physical observables: the ground-state energy, the number of phonons in the polaron cloud, the effective mass, and the isotope exponent on the effective mass. For a given observable $Q$, the expectation value is the statistical average over trajectories at $\mathbf{P}=0$ (ground state), which can be written in the form 


$$
\langle Q\rangle_{0}=\frac{\int_{\mathrm{tw}} \operatorname{Dr} Q w\left(N_{s}\right)}{\int_{\mathrm{tw}} \operatorname{Dr} w\left(N_{s}\right)},
$$

where the phonon degrees of freedom have been integrated out, and $w\left(N_{s}\right)$ is given by Eq. (31). This corresponds to a simple arithmetic average within the QMC simulation.

The ground state $(\mathbf{P}=0)$ energy estimator is given by ${ }^{48}$

$$
E_{0}(0)=-\left\langle\frac{1}{w} \frac{\partial w}{\partial \beta}\right\rangle_{0}=-\left\langle\frac{\partial A}{\partial \beta}\right\rangle_{0}-\frac{1}{\beta}\left\langle\sum_{s} N_{s}\right\rangle_{0},
$$

which follows from the corresponding finite-imaginary-time energy estimator ${ }^{46}$ in the continuum limit. Within the QMC simulation, then, we must gather separate statistics for the total number of kinks $\Sigma_{s} N_{s}$ and the quantity $\partial A / \partial \beta$. One can see that the expression for $\partial A / \partial \beta$ is easily obtained by analytically differentiating Eq. (40) with respect to $\beta$.

The number of phonons in the polaron cloud $N_{\mathrm{ph}}$ quantifies the amount of lattice deformation associated with the polaron. The value of $N_{\mathrm{ph}}$ is given by the expectation value of the phonon number operator, which can be isolated from the model Hamiltonian using the fact that $\partial H /\left.\partial(\hbar \omega)\right|_{\lambda \omega}$ $=\Sigma_{\mathbf{m}} d_{\mathbf{m}}^{\dagger} d_{\mathbf{m}}$. This can be related to the action via the free energy $F_{0}=-\beta^{-1} \ln Z_{0}$ to give

$$
N_{\mathrm{ph}}=\left\langle\sum_{\mathbf{m}} d_{\mathbf{m}}^{\dagger} d_{\mathbf{m}}\right\rangle_{0}=\left.\frac{\partial F_{0}}{\partial(\hbar \omega)}\right|_{\lambda \omega}=-\frac{1}{\bar{\beta}}\left\langle\left.\frac{\partial A}{\partial \bar{\omega}}\right|_{\lambda \bar{\omega}}\right\rangle_{0},
$$

where $\partial A /\left.\partial \bar{\omega}\right|_{\lambda \bar{\omega}}$ is easily obtained by differentiating Eq. (40) with respect to $\bar{\omega}$ holding the product $\lambda \bar{\omega}$ constant.

As discussed in Sec. III A, by imposing twisted boundary conditions in imaginary time, dynamical properties can be inferred directly from QMC simulation. The effective mass of the polaron $\mathrm{m}^{*}$, for the isotropic or one-dimensional case, may be measured using

$$
\frac{m_{0}}{m^{*}}=\frac{1}{2 \bar{\beta}}\left\langle(\Delta \mathbf{r})^{2}\right\rangle_{0}
$$

where the difference in position of the endpoints of the trajectory $\Delta \mathbf{r}=\mathbf{r}(\bar{\beta})-\mathbf{r}(0)$ is measured in units of the lattice constant $a$, and $m_{0}$ is the bare electron mass.

The isotope effect is most often observed via its influence on the superconducting transition temperature $T_{\mathrm{c}}$. The dependence of $T_{\mathrm{c}}$ on the mass of the lattice ions $M$ has been found empirically to be $T_{\mathrm{c}} \propto M^{-\alpha},{ }^{65,66}$ where $\alpha$ is known as the isotope exponent on $T_{\mathrm{c}}$. In a similar way, let us define the isotope exponent on the effective mass $\alpha_{m}{ }^{*}$ as

$$
\alpha_{m^{*}}=\frac{M}{m^{*}} \frac{d m^{*}}{d M}=-M \frac{m^{*}}{m_{0}} \frac{\partial}{\partial M}\left(\frac{m_{0}}{m^{*}}\right)
$$

for the isotropic or one-dimensional case. On substitution of the derivative of $m_{0} / m^{*}$, as in Eq. (50), with respect to $M$, we have

$$
\alpha_{m^{*}}=\frac{\bar{\omega}}{2} \frac{1}{\left\langle(\Delta \mathbf{r})^{2}\right\rangle_{0}}\left[\left\langle(\Delta \mathbf{r})^{2} \frac{\partial A}{\partial \bar{\omega}}\right\rangle_{0}-\left\langle(\Delta \mathbf{r})^{2}\right\rangle_{0}\left\langle\frac{\partial A}{\partial \bar{\omega}}\right\rangle_{0}\right],
$$

where the ionic mass enters our formalism via the phonon frequency $\omega=(k / M)^{1 / 2}$, where $k$ is some "spring constant" and $\partial A / \partial \bar{\omega}$ is easily obtained from Eq. (40). [For a general $D$-dimensional system, we may define the $d$ th component of the isotope exponent on the effective mass as $\alpha_{m^{*}}^{(d)}$ $=-M\left(m_{d}^{*} / m_{0, d}\right) \partial / \partial M\left(m_{0, d} / m_{d}^{*}\right), \quad$ where $\quad m_{0, d} / m_{d}^{*}$ $=(2 \bar{\beta})^{-1}\left\langle\left(\Delta r_{d}\right)^{2}\right\rangle_{0}$, with $\Delta r_{d}=r_{d}(\bar{\beta})-r_{d}(0)$, and thus we may write $\alpha_{m^{*}}^{(d)}$ as Eq. (52) with every $\Delta \mathbf{r}$ replaced by $\Delta r_{d}$.]

\section{Simulation details}

The QMC scheme is based on the simulation of a single trajectory $\mathbf{r}(\bar{\tau})$ in imaginary time $0 \leqslant \bar{\tau} \leqslant \bar{\beta}$. The standard Metropolis algorithm is used to alter the shape of the trajectory by the addition and deletion of single kinks, as described in Sec. IV A. The start of the trajectory $\mathbf{r}(0)$ does not change throughout the simulation, but the other end $\mathbf{r}(\bar{\beta})$ is "free" (open boundary conditions in imaginary time). In practice, the shape of the trajectory was represented using a list containing the imaginary-time, and the direction, of each kink. In addition, we also kept track of the value of $\Delta \mathbf{r}=\mathbf{r}(\bar{\beta})$ $-\mathbf{r}(0)$, and the the total number of kinks of each direction $\left\{N_{s}\right\}$. The major computational task is the evaluation, on each Monte Carlo step, of the action given by Eq. (40). (The number of exponential-function-evaluations was reduced by storing the values of $A_{1}^{(j)}$ and $K^{(j)}$ along with each kink, reducing the overall computational effort.) In order to calculate the expectation values of the observables given in Sec. IV C, separate statistics for the quantities $\left\langle\Sigma_{s} N_{s}\right\rangle_{0},\langle\partial A / \partial \bar{\beta}\rangle_{0}$, $\left\langle\partial A /\left.\partial \bar{\omega}\right|_{\lambda \bar{\omega}}\right\rangle_{0},\left\langle(\Delta \mathbf{r})^{2}\right\rangle_{0},\langle\partial A / \partial \bar{\omega}\rangle_{0}$, and $\left\langle(\Delta \mathbf{r})^{2} \partial A / \partial \bar{\omega}\right\rangle_{0}$ were gathered every 10-50 Monte Carlo steps. We only consider the case of $\mathbf{P}=0$, corresponding to the ground state of the system, where there is no sign problem.

The four one-dimensional interaction models studied differ only in the value of the screening length $R_{\mathrm{sc}}$. The model dependency enters the simulation via $\Phi\left(\mathbf{r}^{\prime}\right)$ given by Eq. (46). For each model, simulations were conducted for various different values of the dimensionless parameters $\bar{\omega}$ and $\lambda$.

The value of $\bar{\beta}$ was set at a sufficiently large value to enforce the low-temperature limit $\exp (\bar{\omega} \bar{\beta}) \rightarrow \infty$. For the present simulations, a value of $\bar{\beta} \geqslant 15$ was found to make the finite-temperature error negligible. (Reducing the value of $\bar{\omega}$, or increasing $\lambda$, beyond those studied here would require this value of $\bar{\beta}$ to be increased.) Increasing the value of $\bar{\beta}$ in- 
creases the "length" of the trajectory (which involves more kinks), and thus increases the computational effort required to perform each Monte Carlo step.

For each set of model parameters $\left(R_{\mathrm{sc}}, \bar{\omega}\right.$, and $\left.\lambda\right)$, between 3 and 6 statistically independent Monte Carlo runs were performed, each using a different value of the inverse temperature $15 \leqslant \bar{\beta} \leqslant 25$. The number of Monte Carlo steps in each run was taken to be about five times the "warm-up" period. Typically, the runs consisted of between $1 \times 10^{7}$ and $5 \times 10^{7}$ steps in total. (The statistics gathered for each set of runs were viewed together graphically, in order to better estimate the point at which equilibrium had been reached.) Only those statistics gathered after the estimated warm-up period were included in the averages. Given that the finitetemperature error is small, and in the absence of systematic finite-size and finite-time-step errors, the main source of error is statistical. The size of the statistical error depends on $f_{\mathbf{m}}(\mathbf{n})$ and $\bar{\beta}$, as well as on $\bar{\omega}$ and $\lambda$. For each set of model parameters $\left(R_{\mathrm{sc}}, \bar{\omega}\right.$, and $\left.\lambda\right)$, we performed a sufficient number of runs to ensure that the Monte Carlo averages were determined to a statistical error of less than $1 \%$.

\section{LIMITING CASES}

\section{A. Strong coupling (small polaron) regime}

When the electron-phonon coupling is strong, the electron becomes "trapped" in a potential well created by the induced lattice distortion. In this case the "size" of the polaron state can become comparable with the lattice constant, and the term "small polaron" is used. The condition for small polaron formation is $\lambda=E_{p} / z t \geqslant 1$, which is referred to as the strong-coupling regime. ${ }^{42}$ The small polaron can move from site to site (at zero temperature) through the action of zeropoint motion.

An analytical method to determine the effective mass and energy dispersion in the strong-coupling regime, for a lattice polaron with a general electron-phonon interaction force $f_{\mathbf{m}}(\mathbf{n}),{ }^{36}$ is based on the Lang-Firsov canonical transformation $^{15}$ (which renders the transformed Hamiltonian diagonal for $\lambda \rightarrow \infty$ ), followed by a second-order perturbation technique that uses $1 / \lambda$ as a small parameter. ${ }^{42}$ For nearest-neighbor hopping only, and forms of $f_{\mathbf{m}}(\mathbf{n})$ that depend on the relative lattice distance $|\mathbf{m}-\mathbf{n}|$, the result for the lowest energy levels reads ${ }^{36}$

$$
E(\mathbf{k})=-E_{p}-\varepsilon_{p}(\mathbf{k})-\sum_{\mathbf{k}^{\prime},\left\{n_{\mathbf{q}}\right\}} \frac{\left|\left\langle\mathbf{k}, 0\left|\sum_{\mathbf{n n}^{\prime}} t_{\mathbf{n n}^{\prime}} \exp \left[\sum_{\mathbf{m} \cdot \mathbf{q}} \frac{\left[f_{\mathbf{m}}(\mathbf{n})-f_{\mathbf{m}}\left(\mathbf{n}^{\prime}\right)\right]\left(e^{i \mathbf{q} \cdot \mathbf{m}} d_{\mathbf{q}}^{\dagger}-e^{-i \mathbf{q} \cdot \mathbf{m}} d_{\mathbf{q}}\right)}{\sqrt{2 N M \hbar \omega_{\mathbf{q}}^{3}}}\right] c_{\mathbf{n}^{\prime}}^{\dagger}, c_{\mathbf{n}}\right| \mathbf{k}^{\prime},\left\{n_{\mathbf{q}}\right\}\right\rangle\right|^{2}}{\hbar \omega \sum_{\mathbf{q}} n_{\mathbf{q}}},
$$

where the summation is over intermediate states with one or more phonons, $\Sigma_{\mathbf{q}} n_{\mathbf{q}}>0$, the polaronic energy-level shift

$$
E_{p}=\frac{1}{2 M \omega^{2}} \sum_{\mathbf{m}} f_{\mathbf{m}}^{2}(0)
$$

(used to define $\lambda$ ) corresponds with the solution for $\lambda \rightarrow \infty$, and the small-polaron dispersion

$$
\varepsilon_{p}(\mathbf{k})=t \sum_{\mathbf{n} \neq 0} e^{-g^{2}(1)} e^{-i \mathbf{k} \cdot \mathbf{n}},
$$

where $g^{2}$ is known as the (zero temperature) mass renormalization exponent, given by

$$
g^{2}(1)=\frac{1}{2 M \hbar \omega^{3}} \sum_{\mathbf{m}}\left[f_{\mathbf{m}}^{2}(0)-f_{\mathbf{m}}(0) f_{\mathbf{m}}(1)\right] .
$$

The final term of Eq. (53) is quadratic with respect to the bare hopping amplitude $t_{\mathbf{n} n^{\prime}}$, and produces a negative correction to the energy. (Here, $n_{\mathbf{q}}$ is the number of phonons with wave vector $\mathbf{q}$, and $\left|\mathbf{k}^{\prime}, n_{\mathbf{q}}\right\rangle$ is an excited state that consists of a single electron with wave vector $\mathbf{k}^{\prime}$ and one or more phonons.) It is of order of $1 / \lambda^{2}$ and is almost independent of k. ${ }^{36}$
In the ground state of the system $(\mathbf{k}=0)$, the value of $\varepsilon_{p}(\mathbf{k})$ is real and small compared with $-E_{p}$, and so Eq. (53) is dominated by $-E_{P}$. Thus, the (dimensionless) strongcoupling result for the ground-state energy is

$$
\frac{E_{0}}{t}=z \lambda,
$$

and since $E_{p}=N_{\mathrm{ph}} \hbar \omega$, it follows that the number of phonons in the polaron cloud at $\mathbf{k}=0$ is given by

$$
N_{\mathrm{ph}}=\frac{z \lambda}{\bar{\omega}} .
$$

Assuming that the third term in Eq. (53) is completely independent of $\mathbf{k}$, then the inverse effective mass, for the isotropic or one-dimensional case, is given by

$$
\frac{m_{0}}{m^{*}}=\left.m_{0} \frac{\partial^{2} E(k)}{\partial k^{2}}\right|_{k \rightarrow 0}=e^{-g^{2}(1)} .
$$

This can be conveniently expressed in dimensionless form as

$$
\frac{m_{0}}{m^{*}}=\exp \left(\frac{-2 \gamma \lambda}{\bar{\omega}}\right),
$$

where we have defined the constant 
TABLE I. Values of the dimensionless parameter $\gamma=1$ $-\Sigma_{\mathbf{m}} f_{\mathbf{m}}(0) f_{\mathbf{m}}(1) / \Sigma_{\mathbf{m}^{\prime}} f_{\mathbf{m}^{\prime}}^{2}(0)$ for the one-dimensional models studied in this work, where $\gamma$ depends only on the shape of the interaction force.

\begin{tabular}{cc}
\hline \hline Interaction model & $\gamma$ \\
\hline Holstein & 1.000 \\
Screened Fröhlich $\left(R_{\mathrm{sc}}=1\right)$ & 0.745 \\
Screened Fröhlich $\left(R_{\mathrm{sc}}=3\right)$ & 0.531 \\
Nonscreened Fröhlich & 0.387 \\
\hline \hline
\end{tabular}

$$
\gamma \equiv 1-\frac{\sum_{\mathbf{m}} f_{\mathbf{m}}(0) f_{\mathbf{m}}(1)}{\sum_{\mathbf{m}^{\prime}} f_{\mathbf{m}^{\prime}}^{2}(0)},
$$

which depends only on the shape of the electron-phonon interaction force. The isotope exponent on the effective mass $\alpha_{m}{ }^{*}$, defined in Eq. (51), can be written in terms of $\omega$ $\propto M^{-1 / 2}$ as

$$
\alpha_{m^{*}}=\frac{m^{*}}{m_{0}} \frac{\omega}{2} \frac{\partial}{\partial \omega}\left(\frac{m_{0}}{m^{*}}\right),
$$

for the isotropic (or one-dimensional) case. Thus, the (dimensionless) strong-coupling isotope exponent is given by ${ }^{37}$

$$
\alpha_{m}=\frac{\gamma \lambda}{\bar{\omega}} .
$$

The value of the dimensionless constant $0 \leqslant \gamma \leqslant 1$ must in general be determined numerically: the value calculated for each of the one-dimensional interaction models studied in this work is presented in Table I.

\section{B. Weak coupling (large polaron) regime}

In 1950 Fröhlich et al. ${ }^{67}$ considered the ground state of a polaron in the weak-coupling limit $\lambda \ll 1$, using second-order perturbation theory, under the condition that there is never more than one phonon virtually excited, and the discreteness of the lattice is unimportant (because the band-electron-like "large polaron" state ${ }^{68}$ is much larger than the lattice constant). In our case we use the tight-binding dispersion

$$
\varepsilon_{0}(\mathbf{k})=-2 t \cos (k a)
$$

rather than the parabolic approximation $\varepsilon_{0}(\mathbf{k})=\hbar^{2} \mathbf{k}^{2} / 2 m_{0}$ $+O\left(\mathbf{k}^{4}\right)$, where $m_{0}=\hbar^{2} /\left(2 t a^{2}\right)$ used by Fröhlich. ${ }^{67}$ Thus we write

$$
H=\varepsilon_{0}(\mathbf{k})+\hbar \omega \sum_{\mathbf{q}} d_{\mathbf{q}}^{\dagger} d_{\mathbf{q}}+H_{e-\mathbf{p h}},
$$

where the electron-phonon term $H_{e \text {-ph }}$ is a small perturbation. Assuming that $f_{\mathbf{m}}(\mathbf{n})$ depends only on the relative lattice distance $|\mathbf{m}-\mathbf{n}|$, then $H_{e \text {-ph }}$ given by Eq. (4) may be written in momentum representation as

$$
H_{e-\mathbf{p h}}=-\sqrt{\tilde{\kappa}} \sum_{\mathbf{q}, \mathbf{k}} \tilde{f}_{\mathbf{q}}\left(c_{\mathbf{k}-\mathbf{q}}^{\dagger} c_{\mathbf{k}} d_{\mathbf{q}}^{\dagger}+\text { H.c. }\right),
$$

where we have defined

$$
\tilde{\kappa}=\frac{z \lambda \bar{\omega} t^{2}}{\sum_{\mathbf{m}} \bar{f}_{\mathbf{m}}^{2}(0)}
$$

and

$$
\tilde{f}_{\mathbf{q}}=\sum_{\mathbf{r}} \bar{f}_{\mathbf{r}}(0) e^{-i \mathbf{q} \cdot \mathbf{r} / \hbar}
$$

Here, the summation is over all values of $\mathbf{r}=\mathbf{m}-\mathbf{n}$ for which $\bar{f}_{\mathbf{m}}(\mathbf{n})=\bar{f}(\mathbf{m}-\mathbf{n})=\bar{f}_{\mathbf{r}}(0)$ is a function of a single variable only. Using standard second-order perturbation theory, with an initial state that consists of an electron of momentum $\hbar \mathbf{k}$ and no phonons, and an intermediate state that consists of an electron with momentum $\hbar(\mathbf{k}-\mathbf{q})$ and a single phonon of momentum $\hbar \mathbf{q}$, the energy measured from the bottom of the electron band is given by

$$
E(\mathbf{k})=\varepsilon_{0}(\mathbf{k})-\widetilde{\kappa} \sum_{\mathbf{q}} \frac{\left|\tilde{f}_{\mathbf{q}}\right|^{2}}{W},
$$

where we have defined

$$
W=\varepsilon_{0}(\mathbf{k}-\mathbf{q})+\hbar \omega-\varepsilon_{0}(\mathbf{k}) .
$$

Here the ground-state energy $E_{0}$ occurs at $\mathbf{k}=\mathbf{0}$ and $\Sigma_{\mathbf{q}}$ is a suitable Brillouin zone average.

The number of virtual phonons in the polaron cloud is defined as $N_{\mathrm{ph}}=\Sigma_{\mathbf{q}}\left\langle 0^{\prime}\left|d_{\mathbf{q}}^{\dagger} d_{\mathbf{q}}\right| 0^{\prime}\right\rangle$, where $\left|0^{\prime}\right\rangle$ represents the eigenstate for the perturbed Hamiltonian. Using standard first-order perturbation theory, this is given by

TABLE II. The numerically calculated values of the coefficient of the linear term in $\lambda$ in Eqs. (74)-(77), for each one-dimensional interaction model at $\bar{\omega}=1$ in the weak coupling limit. For example, the ground-state energy (74) of the Holstein polaron is given by $E_{0} / t=-2-0.894 \lambda$.

\begin{tabular}{cccccc}
\hline \hline Interaction model & $\Sigma_{\mathbf{m}} f_{\mathbf{m}}^{2}(0)$ & $\Upsilon_{E_{0}}$ & $\Upsilon_{N_{\mathrm{ph}}}$ & $\Upsilon_{m_{0} / m^{*}}$ & $\Upsilon_{\alpha_{m^{*}}}$ \\
\hline Holstein $\left(R_{\mathrm{sc}} \rightarrow 0\right)$ & 1.000 & 0.894 & 0.537 & 0.537 & 0.125 \\
Screened Fröhlich $\left(R_{\mathrm{sc}}=1\right)$ & 1.034 & 1.080 & 0.736 & 0.625 & 0.200 \\
Screened Fröhlich $\left(R_{\mathrm{sc}}=3\right)$ & 1.133 & 1.257 & 0.938 & 0.678 & 0.266 \\
Unscreened Fröhlich $\left(R_{\mathrm{sc}} \rightarrow \infty\right)$ & 1.269 & 1.394 & 1.104 & 0.687 & 0.306 \\
\hline \hline
\end{tabular}




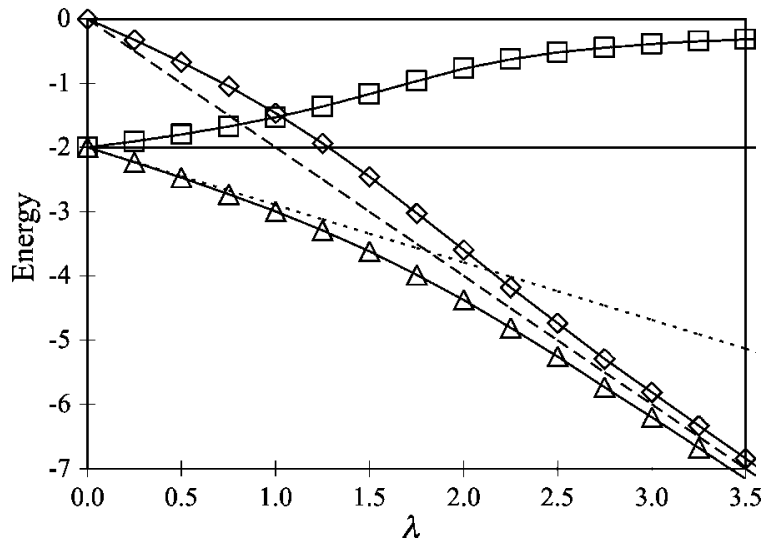

FIG. 4. The variation of ground-state energy $E_{0}(0)$ (triangles), together with potential energy (diamonds) and kinetic energy (squares), with coupling $\lambda$ for the one-dimensional Holstein model with a dimensionless phonon frequency of $\bar{\omega}=1$. The dashed line is the strong coupling perturbation (SCP) result (57) and the dotted line the weak-coupling perturbation (WCP) result (74). One can clearly distinguish the large-polaron, transition and small-polaron regions.

$$
N_{\mathrm{ph}}=\tilde{\kappa} \sum_{\mathbf{q}} \frac{\left|\tilde{f}_{\mathbf{q}}\right|^{2}}{W^{2}}
$$

The effective mass is easily found by differentiating Eq. (69) twice with respect to $\mathbf{k}$, according to Eq. (59), to give

$$
\frac{m_{0}}{m^{*}}=1-\frac{\tilde{\kappa}}{2 a t^{2}} \sum_{\mathbf{q}} \frac{\left|\tilde{f}_{\mathbf{q}}\right|^{2}\left(2 W^{\prime 2}-W^{\prime \prime} W\right)}{W^{3}},
$$

where $W^{\prime}=\partial W / \partial \mathbf{k}$ and $W^{\prime \prime}=\partial^{2} W / \partial \mathbf{k}^{2}$. Differentiating this expression with respect to $\omega$, according to Eq. (62), gives the isotope exponent on the effective mass as

$$
\begin{aligned}
\alpha_{m^{*}}= & \frac{\widetilde{\kappa}}{4 a t^{2}} \frac{m^{*}}{m_{0}} \sum_{\mathbf{q}}\left[\frac{\left|\tilde{f}_{\mathbf{q}}\right|^{2} W^{\prime \prime}(W-2 \hbar \omega)}{W^{3}}\right. \\
& \left.-\frac{\left|\tilde{f}_{\mathbf{q}}\right|^{2} 2 W^{\prime 2}(W-3 \hbar \omega)}{W^{4}}\right] .
\end{aligned}
$$

The above expressions for the observables may be written in the form

$$
\begin{gathered}
\frac{1}{t} E_{0}=-2-\lambda \Upsilon_{E_{0}}(\bar{f}, \bar{\omega}), \\
N_{\mathrm{ph}}=\lambda \Upsilon_{N_{\mathrm{ph}}}(\bar{f}, \bar{\omega}), \\
\frac{m_{0}}{m^{*}}=1-\lambda \Upsilon_{m^{*}}(\bar{f}, \bar{\omega}),
\end{gathered}
$$

and

$$
\alpha_{m^{*}}=\frac{\lambda}{m_{0} / m^{*}} \Upsilon_{\alpha_{m}{ }^{*}}(\bar{f}, \bar{\omega}),
$$

where $Y(\bar{f}, \bar{\omega})$ is the coefficient of the linear term in $\lambda$, which may be calculated numerically. We are interested in the ground-state properties and thus must evaluate the above expressions at $\mathbf{k} \rightarrow 0$. The ground-state values of $\Upsilon(\bar{f}, \bar{\omega})$ for each one-dimensional interaction model are presented in Table II for $\bar{\omega}=1$.

The coefficients can be expressed in closed form for the simplest case of the one-dimensional Holstein interaction. Here we find that the energy is

$$
E(\mathbf{k})=-2 t \cos (k a)-\frac{2 \bar{\omega} t \lambda}{\left\{[\bar{\omega}+2 \cos (k a)]^{2}-4\right\}^{1 / 2}},
$$

so that

$$
\Upsilon_{E_{0}}(\bar{f}, \bar{\omega})=\frac{2 \bar{\omega}}{\left(\bar{\omega}^{2}+4 \bar{\omega}\right)^{1 / 2}}
$$

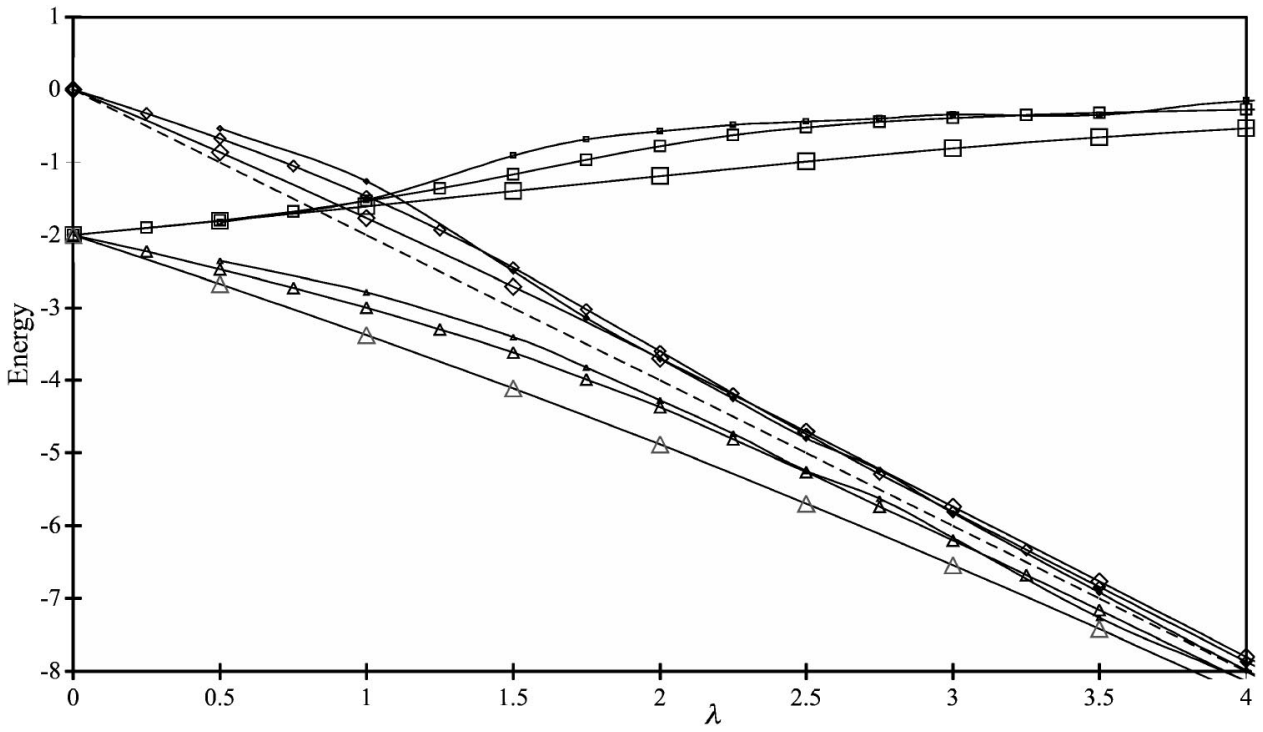

FIG. 5. The variation of ground-state energy $E_{0}(0)$ (triangles), together with potential energy (diamonds) and kinetic energy (squares), with coupling $\lambda$, for the one-dimensional Holstein model with a phonon frequency of $\bar{\omega}=0.5 \quad$ (small symbols), $\quad 1.0$ (medium-size symbols), and 3.0 (large symbols). As $\bar{\omega}$ increases, the transition region shifts to higher $\lambda$ and becomes broader. 
TABLE III. The boundaries of the transition region for the one-dimensional Holstein model at different values of the (dimensionless) phonon frequency $\bar{\omega}$. The estimates are based on the QMC results for the energies and $N_{\text {ph }}$ (observing the changing "trend" in plots of the deviation from the corresponding SCP and WCP results).

\begin{tabular}{ccc}
\hline \hline $\bar{\omega}$ & $\lambda$ (end of large-polaron region) & $\lambda$ (start of small-polaron region) \\
\hline 0.5 & $(1.1 \pm 0.1)$ & $(2.0 \pm 0.2)$ \\
1 & $(1.3 \pm 0.1)$ & $(2.7 \pm 0.3)$ \\
3 & $(2.1 \pm 0.1)$ & $(5.5 \pm 0.6)$ \\
\hline \hline
\end{tabular}

$$
\begin{aligned}
& \Upsilon_{N_{\mathrm{ph}}}(\bar{f}, \bar{\omega})=\frac{2 \bar{\omega}(\bar{\omega}+2)}{\left(\bar{\omega}^{2}+4 \bar{\omega}\right)^{3 / 2}}, \\
& \Upsilon_{m^{*}}(\bar{f}, \bar{\omega})=\frac{2 \bar{\omega}(\bar{\omega}+2)}{\left(\bar{\omega}^{2}+4 \bar{\omega}\right)^{3 / 2}},
\end{aligned}
$$

and

$$
\Upsilon_{\alpha_{m}{ }^{*}}(\bar{f}, \bar{\omega})=\frac{\bar{\omega}^{2}\left(\bar{\omega}^{2}+2 \bar{\omega}+4\right)}{\left(\bar{\omega}^{2}+4 \bar{\omega}\right)^{5 / 2}}
$$

\section{RESULTS}

\section{A. Holstein interaction}

\section{Self-trapping transition}

Let us consider first the QMC results for the simplest case of the one-dimensional Holstein model. The variation of the ground-state energy $E_{0}(0)$ with the coupling constant $\lambda$ is shown in Fig. 4 for a fixed phonon frequency of $\bar{\omega}=1$. The $E_{0}(0)$ curve tends to the weak-coupling perturbation (WCP) result from below as $\lambda \rightarrow 0$, and to the strong-coupling perturbation (SCP) result as $\lambda \rightarrow \infty$. Here we have also plotted the first and second terms of Eq. (48), which provide an indication of the potential energy (PE) and the kinetic energy (KE) of the system, respectively. Three separate regions can be clearly distinguished from Fig. 4 .
(1) The large-polaron region at weak coupling $(\lambda=0$ to $\lambda \approx 1$ in this case) is defined as the range of $\lambda$ for which $E_{0}(0)$ is accurately described by WCP theory (Sec. V B). At $\lambda=0$ the energy is entirely kinetic and represents the bottom of the bare-electron band [with $E_{0}(0)=-z t$ ]. As $\lambda$ increases within this region, $\mathrm{KE}$ increases but remains large compared with PE (nonlocalized bandlike states).

(2) The small-polaron region at strong coupling (in this case $\lambda \approx 2.5$ to $\lambda \rightarrow \infty)$ has $E_{0}(0)$ accurately predicted by SCP theory (Sec. V A). The PE is much greater than the KE (selftrapped states).

(3) The smooth transition region at intermediate coupling ( $\lambda \approx 1$ to $\lambda \approx 2.5$ in this case) between the two regions above. As $\lambda$ increases in this region, a decrease in the PE and an increase in $\mathrm{KE}$ indicates the localization of the polaron.

\section{Variation with phonon frequency}

Now let us consider the way in which the properties of the Holstein polaron are affected by altering the value of the dimensionless phonon frequency $\bar{\omega}$, as defined by Eq. (5). This quantity (also known as the adiabatic ratio) is often used as a parameter in analytical approaches to the polaron problem: the adiabatic regime is defined as the case when $\bar{\omega}<1$, and the antiadiabatic regime as $\bar{\omega}>1$. With this in mind, we present below the properties of the onedimensional Holstein polaron for $\bar{\omega}=0.5, \bar{\omega}=1$, and $\bar{\omega}=3$.

The ground-state energies for all three values of $\bar{\omega}$ are presented together in Fig. 5 against $\lambda$. The PE tends to the

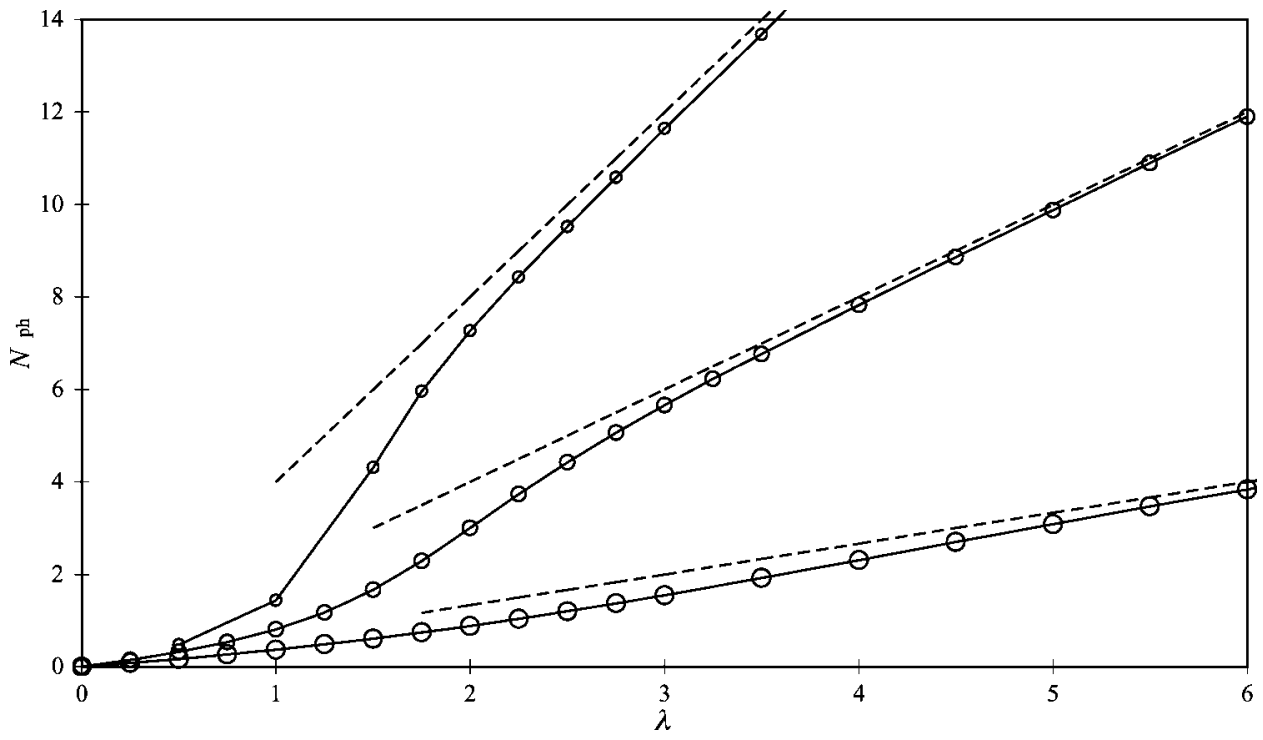

FIG. 6. The number of phonons for the Holstein model at $\bar{\omega}=0.5,1.0,3.0$ (small, mediumsize, and large circles, respectively). The dashed line shows the SCP result (57). The transition region boundaries are the same as those in Fig. 5. 


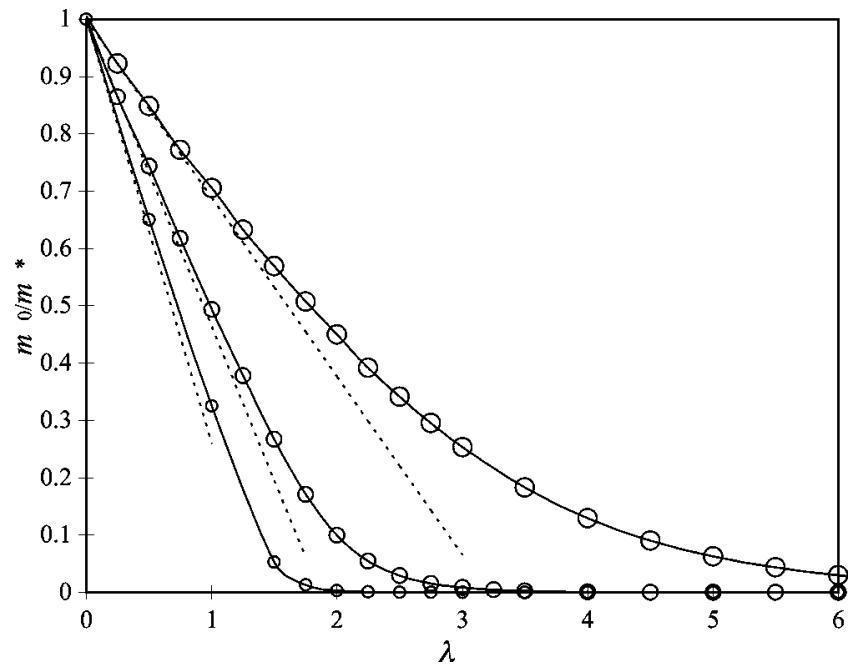

FIG. 7. The inverse effective mass $m_{0} / m^{*}$ (where $m_{0}=\hbar^{2} / 2 t a^{2}$ is the bare-electron mass) for the Holstein model at $\bar{\omega}$ $=0.5,1.0,3.0$ (small, medium-size, and large circles, respectively). The dotted line shows the WCP result (81) for the tight-binding Hamiltonian.

same ( $\bar{\omega}$-independent) SCP result of $E_{0}(0)=2 \lambda$ as $\lambda \rightarrow \infty$. Notice that the KE for intermediate and large values of $\lambda$ decreases as $\bar{\omega}$ increases. This affects the position of the start and end of the transition region. More precisely, as $\bar{\omega}$ increases (1) the start of the smooth transition region moves to higher $\lambda$ and (2) the transition region becomes broader (the start of the small-polaron region is also shifted to larger $\lambda$ ).

The values of $\lambda$ that mark the estimated start and end of the transition region are shown in Table III. The definition of $\lambda$, in Eq. (6), is independent of $\bar{\omega}$, and so naturally characterizes the three regions defined above.

The variation of $N_{\mathrm{ph}}$ with $\lambda$ is presented in Fig. 6 for each value of $\bar{\omega}$. Over all couplings, $N_{\mathrm{ph}}$ decreases as the value of $\bar{\omega}$ increases. Loosely speaking, it is simply "harder" to create phonons of higher frequency. The smooth transition from large to small polaron is again visible in the results for $N_{\mathrm{ph}}$, with the edges of the transition region occurring at the same $\lambda$ as in the above results for $E_{0}(0)$.
The QMC results for the inverse effective mass $m_{0} / \mathrm{m}^{*}$, and the isotope exponent on the effective mass $\alpha_{m}{ }^{*}$, are presented against $\lambda$ in Figs. 7-9, for each value of $\bar{\omega}$. We see from Fig. 7 that increasing $\bar{\omega}$ reduces the effective mass over the entire range of $\lambda$. Both $m_{0} / m^{*}$ and $\alpha_{m}$ tend to the WCP solution as $\lambda$ becomes small, and to the SCP result as $\lambda$ becomes large. However, the results for $\ln \left(\mathrm{m}^{*} / \mathrm{m}_{0}\right)$ tend to the SCP solution at a slower rate than for the other observables.

From all the results for the Holstein model above, we observe that as the value of $\bar{\omega}$ increases, the curve for each observable (PE, $N_{\mathrm{ph}}, m^{*}$, and $\alpha_{m^{*}}$ ) against $\lambda$ moves closer to the line representing the SCP result over the entire range of $\lambda$. In other words, the SCP prediction becomes more applicable as $\bar{\omega}$ increases.

\section{B. Screened Fröhlich interaction}

Here we consider the case of the one-dimensional screened Fröhlich model, Eq. (8), at a fixed dimensionless phonon frequency of $\bar{\omega}=1$. We investigate the way the polaron properties depend on the range of the electron-phonon interaction by comparing the QMC results at four different values of the screening length (shown in Fig. 2): $R_{\mathrm{sc}} \rightarrow 0$ (the short-range Holstein interaction), $R_{\mathrm{sc}}=1, R_{\mathrm{sc}}=3$, and $R_{\mathrm{sc}}$ $\rightarrow \infty$ (the nonscreened Fröhlich interaction).

The QMC results for the energy $\left[E_{0}(0), \mathrm{PE}\right.$, and $\left.\mathrm{KE}\right]$ and the number of phonons in the polaron cloud $N_{\mathrm{ph}}$ are presented against $\lambda$ in Figs. 10 and 11, respectively. They are in excellent agreement with the WCP results at small $\lambda$ (not shown), and tend to the same (that is, $\gamma$-independent) SCP result as $\lambda \rightarrow \infty$. One can see that, as the value of $R_{\mathrm{sc}}$ increases, the KE increases less rapidly with $\lambda$, and so (1) the start of the transition region shifts to higher $\lambda$ and (2) the transition region becomes broader (in $\lambda$ ). This behavior is similar to that found for the Holstein model with increasing $\bar{\omega}$. In the present case, with the phonon frequency $\bar{\omega}$ fixed, the above effect is due only to changing the shape of the electron-phonon interaction force $f_{\mathbf{m}}(\mathbf{n})$. Table IV shows the values of $\lambda$ that mark the estimated start and end of the

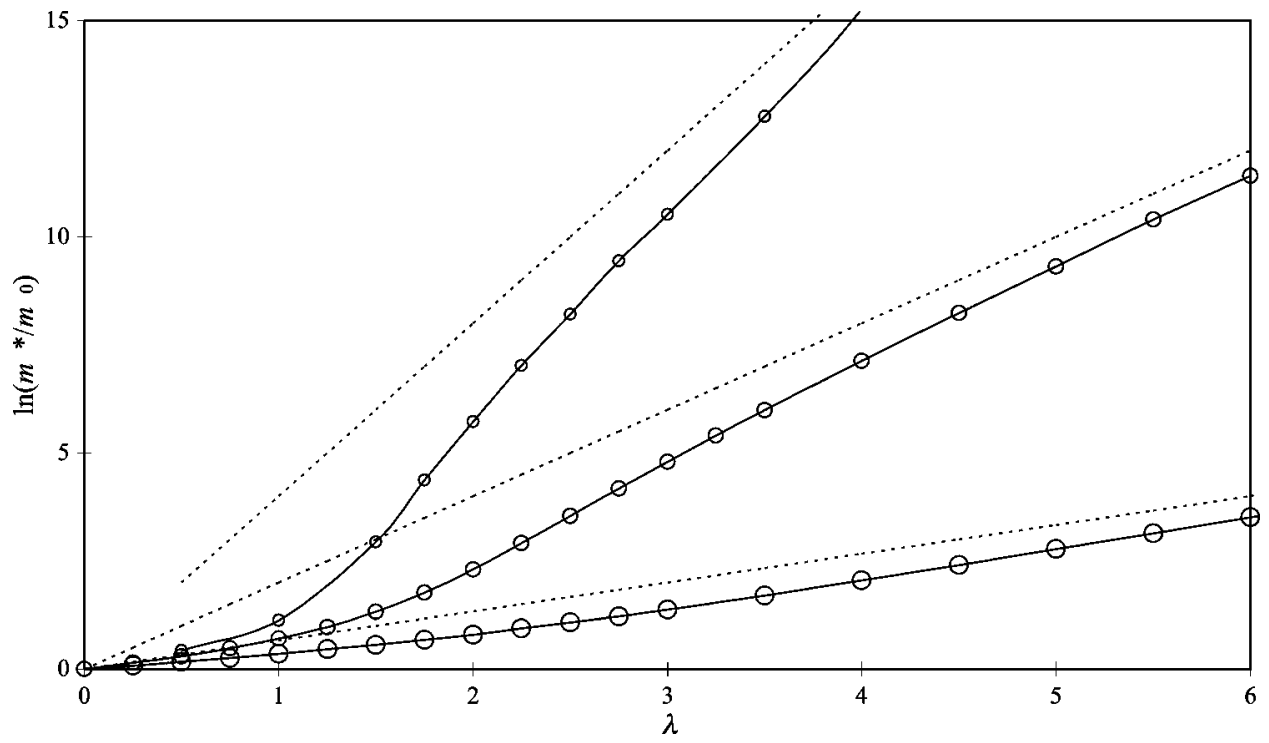

FIG. 8. The logarithm of the effective mass for the Holstein model at $\bar{\omega}=0.5,1.0$, and 3.0 (small, medium-size, and large circles, respectively). The dashed line shows the SCP result $\ln \left(m^{*} / m_{0}\right)=2 \lambda / \bar{\omega}$. 


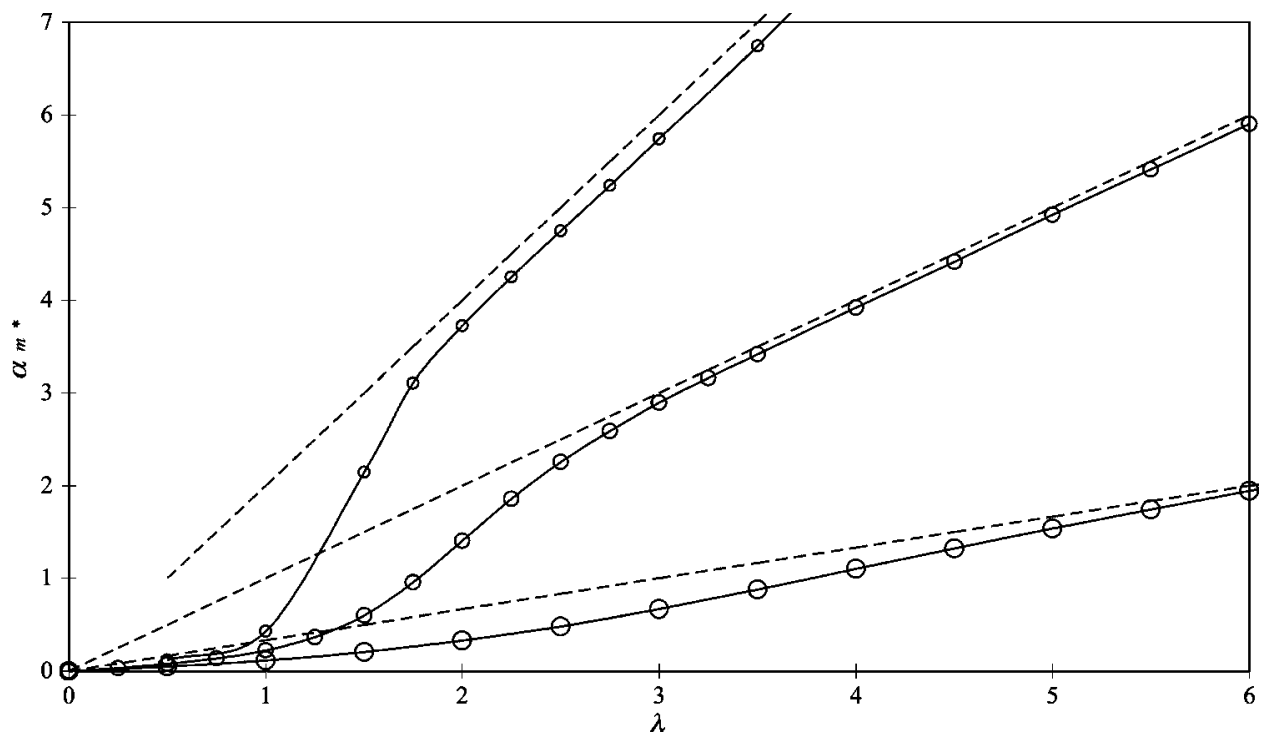

FIG. 9. The isotope exponent for the Holstein model at $\bar{\omega}$ $=0.5,1.0,3.0$ (small, mediumsize, and large circles, respectively). The dotted line shows the SCP result.

transition region for each value of $R_{\mathrm{sc}}$. From the $N_{\mathrm{ph}}$ results in Fig. 11, we notice that increasing the value of $R_{\mathrm{sc}}$ has the effect of increasing $N_{\mathrm{ph}}$ at small $\lambda$, and the opposite effect of decreasing $N_{\mathrm{ph}}$ at large $\lambda$. That is, the order that $N_{\mathrm{ph}}$ appears (with increasing $R_{\mathrm{sc}}$ ) in the large polaron region is the reverse of the order in the small polaron region.

The results for the effective mass are presented in Figs. 12 and 13, and the isotope exponent results in Fig. 14, against $\lambda$ for the same four values of $R_{\mathrm{sc}}$. We can see that for each value of $R_{\mathrm{sc}}$ the QMC results tend to the "model dependent" SCP result [of $\ln \left(m^{*} / m_{0}\right)=2 \gamma \lambda / \bar{\omega}$ and $\alpha_{m}{ }^{*}=\gamma \lambda / \bar{\omega}$, where the value of the mass enhancement factor $\gamma$ is given in Table I for each $R_{\mathrm{sc}}$. This model dependency is in contrast to the above results for $E_{0}(0)$ and $N_{\mathrm{ph}}$, which are $\gamma$ independent in the limit $\lambda \rightarrow \infty$.

An important observation is evident from the plot of $\ln \left(m^{*} / m_{0}\right)$ against $\lambda$ shown in Fig. 13. At intermediate and large couplings (that is, in the transition and small polaron regions), altering the value of $R_{\mathrm{sc}}$ has a dramatic effect on the effective mass. For example, the nonscreened Fröhlich polaron is over $10^{3}$ times "lighter" than the Holstein polaron at $\lambda=4$, and over $10^{4}$ times lighter at $\lambda=5$.

The isotope exponent $\alpha_{m}$ in Fig. 14 shows a strong dependence on the range of electron-phonon interaction (as well as on $\bar{\omega}$ and $\lambda$ ) in the (physically most realistic) intermediate values of coupling. This is important, as experimental measurement (by Zhao et al.) of the exponent of the isotope exponent on the effective supercarrier mass along the $\mathrm{CuO}_{2}$ planes $m_{a b}^{*}$, in the material $\mathrm{La}_{2-x} \mathrm{Sr}_{x} \mathrm{CuO}_{4}$ shows a large value of $\alpha_{m^{*}}^{(a b)}=1.9(2)$ in the deeply underdoped regime $(x=0.06)$, and a much smaller value of $\alpha_{m^{*}}^{(a b)}=0.46(5)$ for optimal doping $(x=0.15) .{ }^{10,69}$ Both the magnitude and radius of the electron-phonon interaction should decrease with doping due to screening. These experimental results show that the former effect is more significant.

Now let us consider the large-polaron region. The QMC results for $m^{*}$ and $\alpha_{m}$ tend to the WCP results for all $R_{\mathrm{sc}}$ (not shown) as $\lambda \rightarrow 0$. As was the case for $N_{\mathrm{ph}}$, we see from Figs. 12 and 14 that the order that $m^{*}$ and $\alpha_{m}{ }^{*}$ appear in the large polaron region (with increasing $R_{\mathrm{sc}}$ ) is the opposite to that in the small polaron region. As can be seen in Fig. 12, the decrease of $m_{0} / m^{*}$ with $\lambda$ is approximately linear for the Holstein model, and approximately exponential for the other screening lengths. In fact, the effective mass of the Fröhlich large-polaron is larger (up to approximately 10\%) than the effective mass of the (short-range) Holstein large polaron.

It is apparent from the above results for the screened Fröhlich model that as $R_{\mathrm{sc}}$ increases (from Holstein to Fröhlich), the QMC results move, in general, closer to the SCP prediction over the entire range of $\lambda$. That is, the SCP prediction becomes generally more applicable as the range of interaction increases (as well as with increasing $\bar{\omega}$ ). This fact allows us to define the intermediate region of the coupling strength and of the adiabatic ratio as a "small polaron" regime for any realistic-range $e$-ph interaction, that is the re-

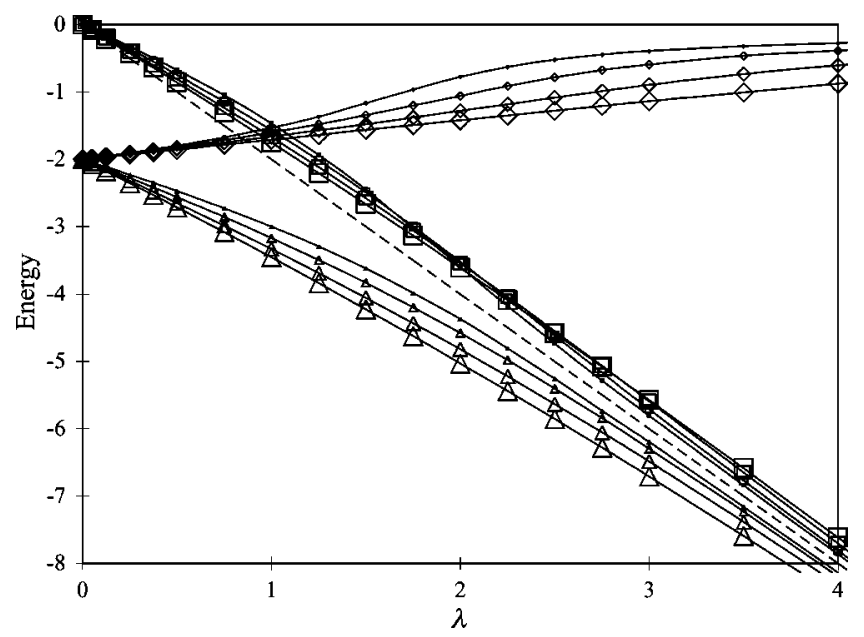

FIG. 10. The ground-state energy $E_{0}(0)$ (triangles), potential energy (squares), and kinetic energy (diamonds) of the onedimensional screened Fröhlich model at $\bar{\omega}=1$, vs $\lambda$, for screening lengths $R_{\mathrm{sc}}=0,1,3, \infty$ (increasing size of symbols). The curves for $E_{0}(0)$ (and $\mathrm{PE}$ ) tends to the same strong coupling perturbation (SCP) result of $E_{0} / t=-2 \lambda$ (dashed line) as $\lambda \rightarrow \infty$. Note the crossing of the potential energy curves near $\lambda=2$. 


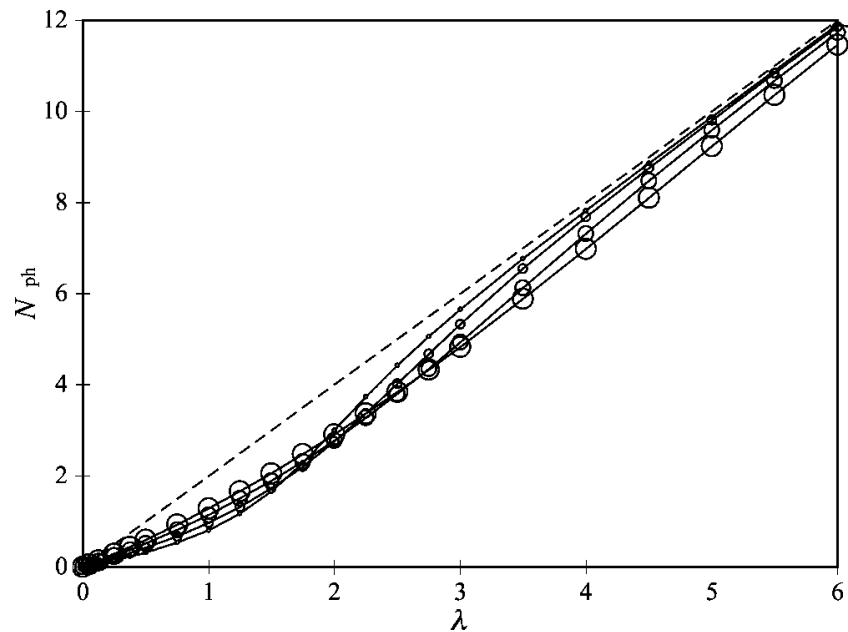

FIG. 11. The number of phonons in the polaron cloud $N_{\text {ph }}$ vs $\lambda$ for screening lengths $R_{\mathrm{sc}}=0,1,3, \infty$ (increasing size of circles) at $\bar{\omega}=1$. The curves tend to the same SCP result of $N_{\mathrm{ph}}=z \lambda / \bar{\omega}$ (dashed line) as $\lambda \rightarrow \infty$.

gime which is well described by the small polaron theory based on the Lang-Firsov transformed Hamiltonian averaged over phonons.

\section{Comparison with other approaches}

As a test of our method, we compare our results with those of other authors. Our main and original computations are for finite values of $R$; however, the majority of published work on lattice polarons relates to the Holstein $(R=0)$ interaction. Figure 15 compares our ground-state energy for $\bar{\omega}$ $=1.0, R=0$ (compare Fig. 4) with that obtained by other authors. To highlight the differences, the weak coupling approximation (74) is subtracted from the energy. The figure compares our results with the QMC data of Hohenadler et $a l .{ }^{49}$ (based on a bosonic path integral, evaluated at inverse temperature $\bar{\beta}=10$ ) and exact diagonalization results for small clusters. ${ }^{70}$ Different computations yield similar values of the ground-state energy, but our QMC energies are closer to the the exact diagonalization results; our calculations have been carried out at a lower temperature $\bar{\beta}=25$. Figure 16 shows good agreement between our results for the effective mass (compare Fig. 8) and variational calculations of Bonča et $a l .{ }^{35}$ (Note that the $\lambda$ in that work corresponds to $\sqrt{2 \omega t \lambda}$ in our notation.) We also reinforce earlier conclusions on the dependence of effective mass with interaction range ${ }^{36,56}$ by interpolation between the Holstein and Fröhlich limits.

\section{CONCLUSIONS}

The general aim of this work was to investigate the way in which the range of electron-phonon interaction governs the physical properties of the (single) lattice polaron. The understanding of this is of considerable current interest because of the increasing amount of experimental evidence suggesting that polarons are present in high-temperature superconducting and colossal magnetoresistance materials.

Perturbation approaches are used in the limits of strong and weak electron-phonon coupling strength $\lambda$. However, in general these do not provide an acceptable description in the (physically most realistic) intermediate coupling range $\lambda$ $\approx 1$. We have performed an extensive Monte Carlo study of the ground state properties for the screened Fröhlich polaron, Eq. (8), in one dimension, over a wide range of coupling.

We have used path-integral quantum Monte Carlo, in which the phonon degrees of freedom are analytically integrated out, leaving only the electron coordinates to be simulated. The use of a path integral with twisted boundary conditions allowed us to extract dynamic properties directly from the simulations. There were no (systematic) errors due to finite size or finite time-step.

The properties measured were the ground state energy $E_{0}(0)$, the number of phonons in the polaron cloud $N_{\mathrm{ph}}$, the effective mass $m^{*}$, and the isotope exponent on the effective mass $\alpha_{m}{ }^{*}$. The QMC results were always found to tend to the weak-coupling perturbation (WCP) predictions for $\lambda \rightarrow 0$, and to the strong-coupling perturbation (SCP) predictions for $\lambda \rightarrow \infty$.

The screened Fröhlich polaron was studied for various values of the screening length $R_{\mathrm{sc}}$ (which essentially controls the range of the electron-phonon interaction): $R_{\mathrm{sc}} \rightarrow 0$ (onsite, Holstein interaction), $R_{\mathrm{sc}}=1, R_{\mathrm{sc}}=3$, and $R_{\mathrm{sc}} \rightarrow \infty$ (longrange, nonscreened Fröhlich interaction). For each value of $R_{\text {sc }}$, we determined the variation of the above observables with $\lambda$, at a fixed phonon frequency of $\bar{\omega}=1$. The main findings are summarized below.

(1) We observe the presence of a self-trapping transition for all values of $R_{\mathrm{sc}}$. In each case, the following three regions are identified.

(a) The large-polaron region at weak coupling, in which the behavior of the system is accurately described by WCP theory. This region is characterized by delocalized, bandelectron-like states.

TABLE IV. The boundaries of the transition region for the one-dimensional screened Fröhlich interaction, at various screening lengths $R_{\mathrm{sc}}$ (measured in units of the lattice constant). The estimates are based on the QMC results for the energy and $N_{\mathrm{ph}}$.

\begin{tabular}{ccc}
\hline \hline Interaction model & $\lambda$ (start of transition) & $\lambda$ (end of transition) \\
\hline Holstein $\left(R_{\mathrm{sc}} \rightarrow 0\right)$ & $(1.1 \pm 0.1)$ & $(2.0 \pm 0.2)$ \\
Screened Fröhlich $\left(R_{\mathrm{sc}}=1\right)$ & $(1.7 \pm 0.1)$ & $(3.8 \pm 0.4)$ \\
Screened Fröhlich $\left(R_{\mathrm{sc}}=3\right)$ & $(2.2 \pm 0.1)$ & $(4.7 \pm 0.4)$ \\
Non-screened Fröhlich $\left(R_{\mathrm{sc}} \rightarrow \infty\right)$ & $(2.9 \pm 0.2)$ & $(6.3 \pm 0.5)$ \\
\hline \hline
\end{tabular}



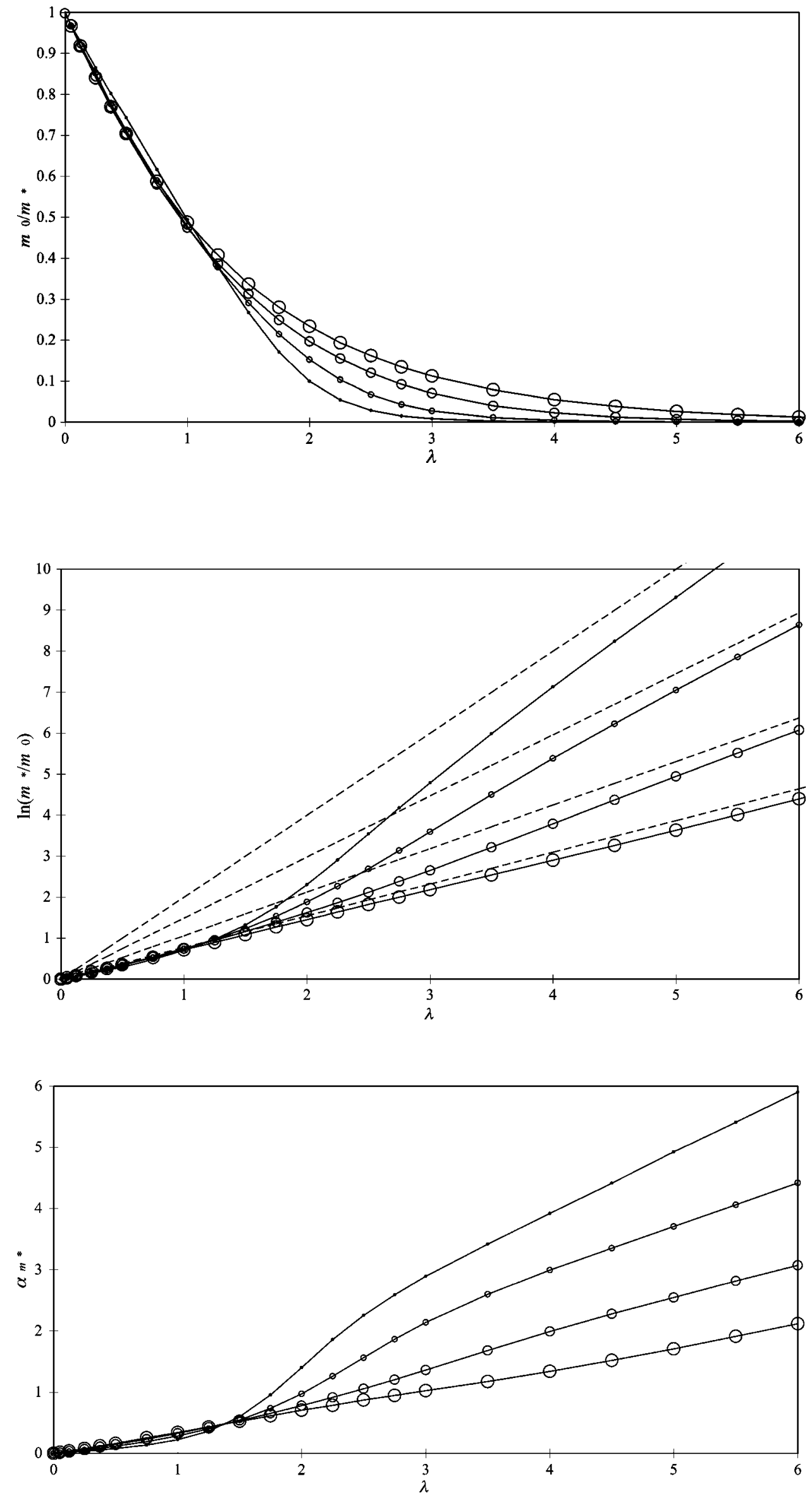

FIG. 12. The inverse effective mass $m_{0} / \mathrm{m}^{*}$ for screening lengths $R_{\mathrm{sc}}=0,1,3, \infty$ (increasing size of circles) vs $\lambda$ at fixed $\bar{\omega}=1$. For weak coupling $(\lambda<1)$ the Holstein large-polaron has a slightly smaller $m^{*}$ than the long-range interactions.
FIG. 13. The logarithm of the effective mass for screening lengths $R_{\mathrm{sc}}=0,1,3, \infty$ (increasing size of circles) vs $\lambda$ at $\bar{\omega}=1$. At intermediate and strong coupling, decreasing the value of $R_{\mathrm{sc}}$ dramatically increases the effective mass. The curves tend to the SCP result (dashed lines) at a slower rate than $E_{0}(0)$ and $N_{\mathrm{ph}}$.
FIG. 14. The isotope exponent on the effective mass $\alpha_{m}{ }^{*}$ for screening lengths $R_{\mathrm{sc}}=0,1,3, \infty$ (increasing size of circles) vs $\lambda$ at $\bar{\omega}=1$. 


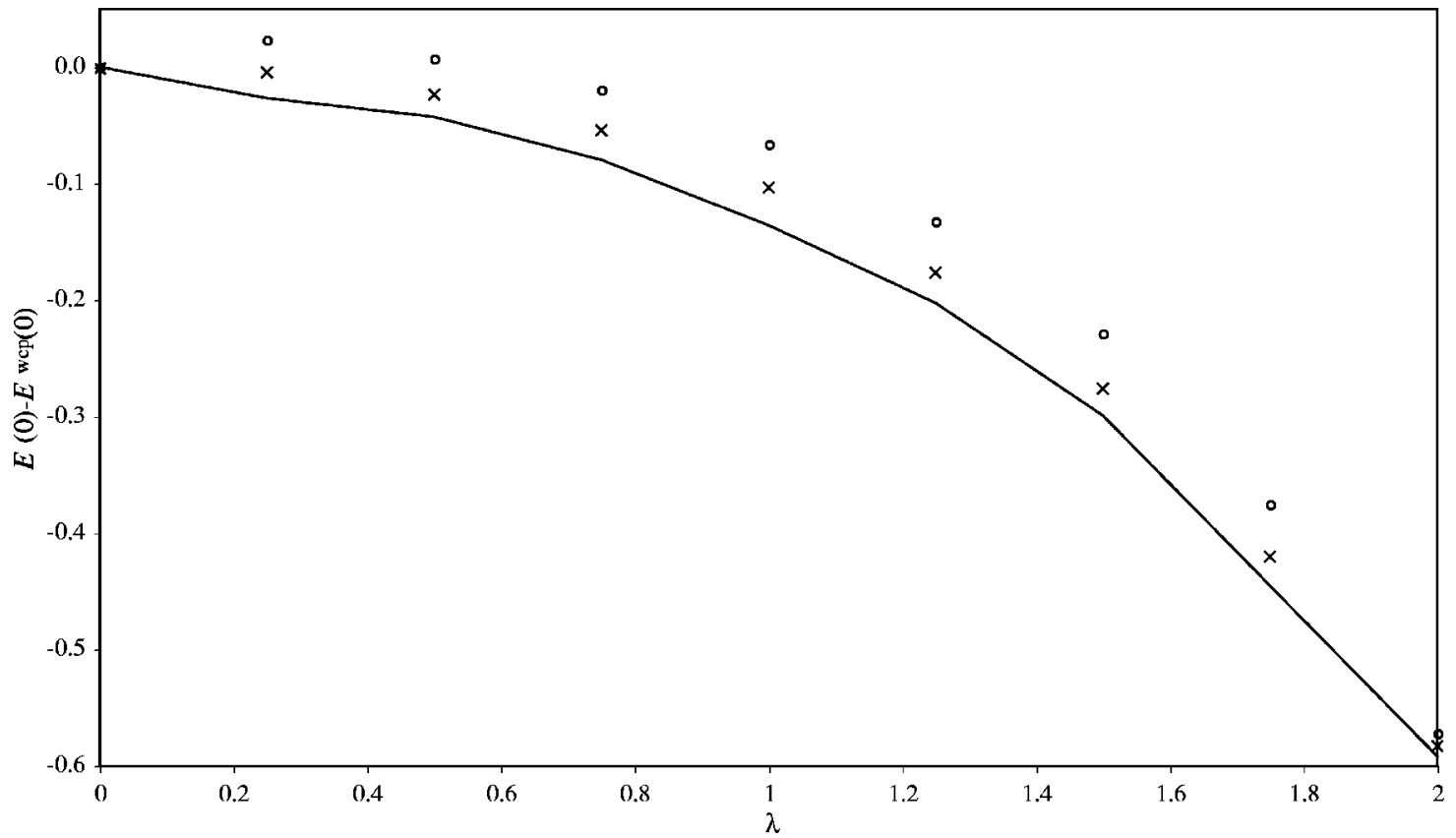

FIG. 15. One-dimensional Holstein polaron ground-state energy for $\bar{\omega}=1.0, R=0$ (crosses) compared with bosonic QMC (circles, Ref. 49) and exact diagonalization energies (solid line, Ref. 70) at $\bar{\omega}=1.0$. The energy in weak-coupling perturbation theory (74) has been subtracted.

(b) The small-polaron region at strong coupling, in which the behavior is accurately described by SCP theory. This region is characterized by localized ("self-trapped") polaronic states.

(c) The transition region between the two, at intermediate coupling. We observe a smooth crossover from large to small polaron in all the observables measured.

(2) The transition region boundaries depend on the range of interaction. As the value of $R_{\mathrm{sc}}$ increases we find the following.

(a) The start of the transition region (the point at which it becomes energetically favorable for localized states to exist) shifts to higher $\lambda$.

(b) The transition region becomes broader (the start of the small polaron region also shifts to higher $\lambda$ ). The small- polaron region starts when the kinetic energy is much smaller than the potential energy.

(c) The values of the observables (PE, $N_{\mathrm{ph}}, m^{*}$, and $\alpha_{m^{*}}$ ) generally move closer to the corresponding SCP result over the entire range of $\lambda$.

(3) In the large polaron region, the effective mass for long-range electron-phonon interaction $\left(R_{\mathrm{sc}}>1\right)$ is found to be up to approximately $10 \%$ larger than that for the Holstein interaction $\left(R_{\mathrm{sc}} \rightarrow 0\right)$.

(4) We observe large variations in the isotope exponent on the effective mass $\alpha_{m}$ in the (physically most realistic) intermediate coupling regime (with changing $R_{\mathrm{sc}}$ and $\lambda$, as well as $\bar{\omega})$. This is encouraging, as experimental observation shows large variations in the isotope exponent with the level of doping in high- $T_{\mathrm{c}}$ materials.

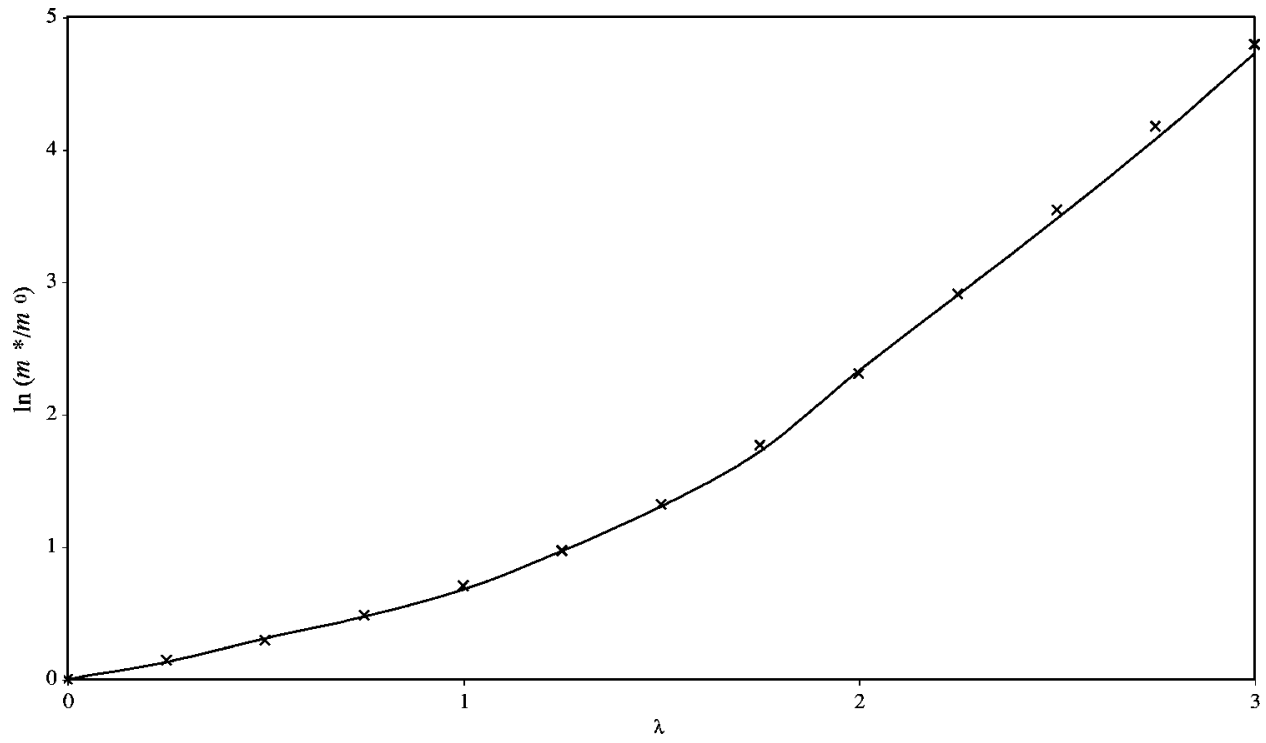

FIG. 16. One-dimensional Holstein polaron effective mass for $\bar{\omega}=1.0, R=0$ (crosses) compared with variational results (solid line, Ref. 35). 
(5) Reducing the range of electron-phonon interaction dramatically increases the effective mass for intermediate and large values of coupling (that is, in the transition and small-polaron regions). In comparison, $E_{0}(0)$ and $N_{\text {ph }}$ are only slightly affected by altering $R_{\mathrm{sc}}$.

(6) We also study the dependence on phonon frequency $\bar{\omega}$ of the Holstein polaron $\left(R_{\mathrm{sc}} \rightarrow 0\right)$. As $\bar{\omega}$ increases we find the following.

(a) The transition region shifts to higher $\lambda$.

(b) The transition region becomes broader.

(c) The observables (PE, $N_{\mathrm{ph}}, m^{*}$, and $\alpha_{m}{ }^{*}$ ) move toward the corresponding SCP result over the entire range of $\lambda$.

(7) Increasing interaction range has a qualitatively similar effect to increasing phonon frequency; compare for example Figs. 8 and 13 or Figs. 9 and 14.

One can also calculate the isotope effect on the whole polaron band dispersion applying the continuous-time quantum Monte-Carlo algorithm. ${ }^{71}$ To deal with the electron spectral function and high-energy excitations, involving phonon shake-off, measured in ARPES, ${ }^{39-41}$ the QMC algorithm has to include the off-diagonal paths, which remains a challenging but solvable problem of our QMC simulations in the site representation. Also other methods as the momentum based QMC, ${ }^{19}$ the numerical diagonalization of vibrating clusters or the renormalisation group are able to calculate the spectral function. The method used here relies on a phonon gap, the errors being exponentially small in $\beta \hbar \omega$, and is therefore more accurate for intermediate and large values of $\omega$. While lower $\omega$ can be also simulated without major difficulty but with increased inverse temperature and therefore increased CPU time, the parameters used cover the most physically relevant range. ${ }^{3}$

\section{ACKNOWLEDGMENTS}

P.E.S. acknowledges support from EPSRC and A.S.A. acknowledges support of the Leverhulme Trust (London).
*Electronic address: j.h.samson@lboro.ac.uk

†Electronic address: pavel.kornilovich@hp.com

Electronic address: a.s.alexandrov@lboro.ac.uk

${ }^{1}$ H. Böttger and V. V. Bryksin, Hopping Conduction in Solids (Academie-Verlag, Berlin, 1985).

${ }^{2}$ G. D. Mahan, Many Particle Physics (Plenum, New York, 1990).

${ }^{3}$ A. S. Alexandrov and N. F. Mott, Rep. Prog. Phys. 57, 1197 (1994); Polarons and Bipolarons (World Scientific, Singapore, 1995).

${ }^{4}$ J. T. Devreese, in Encyclopedia of Applied Physics (VCH, Weinheim, 1996).

${ }^{5}$ In Anharmonic Properties of High- $T_{c}$ Cuprates, edited by D. Mihailović et al. (World Scientific, Singapore, 1995).

${ }^{6}$ In Polarons and Bipolarons in High-T $T_{c}$ Superconductors and Related Materials, edited by E. K. H. Salje, A. S. Alexandrov, and W. Y. Liang (Cambridge University Press, Cambridge, U.K., 1995), p. 353.

${ }^{7}$ A. S. Alexandrov and V. V. Kabanov, Phys. Rev. B 54, 3655 (1996).

${ }^{8}$ A. J. Millis, P. B. Littlewood, and B. I. Shraiman, Phys. Rev. Lett. 74, 5144 (1995).

${ }^{9}$ A. S. Alexandrov and A. M. Bratkovsky, Phys. Rev. Lett. 82, 141 (1999).

${ }^{10}$ G. M. Zhao and D. E. Morris, Phys. Rev. B 51, R16487 (1995); G.-M. Zhao, M. B. Hunt, H. Keller, and K. A. Müller, Nature (London) 385, 236 (1997); R. Khasanov, D. G. Eshchenko, H. Luetkens, E. Morenzoni, T. Prokscha, A. Suter, N. Garifianov, M. Mali, J. Roos, K. Conder, and H. Keller, Phys. Rev. Lett. 92, 057602 (2004).

${ }^{11}$ A. S. Alexandrov, Phys. Rev. B 61, 12315 (2000).

${ }^{12}$ A. B. Migdal, Zh. Eksp. Teor. Fiz. 34, 1438 (1958) [Sov. Phys. JETP 7, 996 (1958)].

${ }^{13}$ G. M. Eliashberg, Zh. Eksp. Teor. Fiz. 38, 966 (1960) [Sov. Phys. JETP 11, 696 (1960)]; 39, 1437 (1960); 12, 1000 (1960).

${ }^{14}$ D. J. Scalapino, in Superconductivity edited by R. D. Parks (Dekker, New York, 1969), p. 449.
${ }^{15}$ I. G. Lang and Yu. A. Firsov, Zh. Eksp. Teor. Fiz. 43, 1843 (1962) [Sov. Phys. JETP 16, 1301 (1963)].

${ }^{16}$ A. S. Alexandrov, Zh. Fiz. Khim. 57, 273 (1983) [Russ. J. Phys. Chem. 57, 167 (1983)].

${ }^{17}$ A. S. Alexandrov and E. A. Mazur, Zh. Eksp. Teor. Fiz. 96, 1773 (1989).

${ }^{18}$ A. S. Alexandrov, Phys. Rev. B 46, 2838 (1992).

${ }^{19}$ N. V. Prokof'ev, B. V. Svistunov, and I. S. Tupitsyn, JETP Lett. 64, 911 (1996); N. V. Prokof'ev, B. V. Svistunov, and I. S. Tupitsyn, JETP 87, 310 (1998); N. V. Prokof'ev and B. V. Svistunov, Phys. Rev. Lett. 81, 2514 (1998); A. S. Mishchenko, N. V. Prokof'ev, A. Sakamoto, and B. V. Svistunov, Phys. Rev. B 62, 6317 (2000); A. S. Mishchenko, N. Nagaosa, N. V. Prokof'ev, A. Sakamoto, and B. V. Svistunov, Phys. Rev. Lett. 91, 236401 (2003).

${ }^{20}$ L. D. Landau, Phys. Z. Sowjetunion 3, 664 (1933).

${ }^{21}$ A. S. Alexandrov, Europhys. Lett. 56, 92 (2001).

${ }^{22}$ V. V. Kabanov and O. Yu. Mashtakov, Phys. Rev. B 47, 6060 (1993).

${ }^{23}$ A. S. Alexandrov, V. V. Kabanov, and D. K. Ray, Phys. Rev. B 49, 9915 (1994).

${ }^{24}$ A. R. Bishop and M. Salkola, in Polarons and Bipolarons in High- $T_{\mathrm{c}}$ Superconductors and Related Materials, edited by E. K. H. Salje, A. S. Alexandrov, and W. Y. Liang (Cambridge University Press, Cambridge, U.K., 1995), p. 353.

${ }^{25}$ H. Fehske, H. Röder, G. Wellein, and A. Mistriotis, Phys. Rev. B 51, 16582 (1995); G. Wellein, H. Röder, and H. Fehske, ibid. 53, 9666 (1996).

${ }^{26}$ F. Marsiglio, Physica C 244, 21 (1995).

${ }^{27}$ Y. Takada and T. Higuchi, Phys. Rev. B 52, 12720 (1995).

${ }^{28}$ H. Fehske, J. Loos, and G. Wellein, Z. Phys. B: Condens. Matter 104, 619 (1997).

${ }^{29}$ T. Hotta and Y. Takada, Phys. Rev. B 56, 13916 (1997).

${ }^{30}$ A. H. Romero, D. W. Brown, and K. Lindenberg, J. Chem. Phys. 109, 6504 (1998); Phys. Rev. B 59, 13728 (1999); 60, 4618 (1999); 60, 14080 (1999). 
${ }^{31}$ A. La Magna and R. Pucci, Phys. Rev. B 53, 8449 (1996).

${ }^{32}$ P. Benedetti and R. Zeyher, Phys. Rev. B 58, 14320 (1998).

${ }^{33}$ T. Frank and M. Wagner, Phys. Rev. B 60, 3252 (1999).

${ }^{34}$ L. Proville and S. Aubry, Eur. Phys. J. B 11, 41 (1999).

${ }^{35}$ J. Bonča, S. A. Trugman, and I. Batistić, Phys. Rev. B 60, 1633 (1999); J. Bonča, T. Katrasnik, and S. A. Trugman, Phys. Rev. Lett. 84, 3153 (2000); L.-C. Ku, S. A. Trugman, and J. Bonča, Phys. Rev. B 65, 174306 (2002); S. El Shawish, J. Bonča, L.-C. $\mathrm{Ku}$, and S. A. Trugman, ibid. 67, 014301 (2003).

${ }^{36}$ A. S. Alexandrov and P. E. Kornilovitch, Phys. Rev. Lett. 82, 807 (1999).

${ }^{37}$ A. S. Alexandrov, Phys. Rev. B 46, R14932 (1992).

${ }^{38}$ A. S. Alexandrov, G.-M. Zhao, H. Keller, B. Lorenz, Y. S. Wang, and C. W. Chu, Phys. Rev. B 64, 140404(R) (2001).

${ }^{39}$ A. Lanzara, P. V. Bogdanov, X. J. Zhou, S. A. Kellar, D. L. Feng, E. D. Lu, T. Yoshida, H. Eisaki, A. Fujimori, K. Kishio, J. I. Shimoyana, T. Noda, S. Uchida, Z. Hussain, and Z. X. Shen, Nature (London) 412, 510 (2001).

${ }^{40}$ X. J. Zhou, J. Shi, T. Yoshida, T. Cuk, W. L. Yang, V. Brouet, J. Nakamura, N. Mannella, S. Komiya, Y. Ando, F. Zhou, W. X. Ti, J. W. Xiong, Z. X. Zhao, T. Sasagawa, T. Kakeshita, H. Eisaki, S. Uchida, A. Fujimori, Z.-Y. Zhang, E. W. Plummer, R. B. Laughlin, Z. Hussain, and Z.-X. Shen, cond-mat/0405130 (unpublished).

${ }^{41}$ G.-H. Gweon, T. Sasagawa, S. Y. Zhou, J. Craf, H. Takagi, D.-H. Lee, and A. Lanzara, Nature (London) 430, 187 (2004).

${ }^{42}$ A. S. Alexandrov, in Theory of Superconductivity: From Weak to Strong Coupling (IOP, Bristol, 2003), pp. 95-158.

${ }^{43}$ R. Blankenbecler, D. J. Scalapino, and R. L. Sugar, Phys. Rev. D 24, 2278 (1981); D. J. Scalapino and R. L. Sugar, Phys. Rev. B 24, 4295 (1981).

${ }^{44}$ J. E. Hirsch, D. J. Scalapino, R. L. Sugar, and R. Blankenbecler, Phys. Rev. Lett. 47, 1628 (1981); J. E. Hirsch, R. L. Sugar, D. J. Scalapino, and R. Blankenbecler, Phys. Rev. B 26, 5033 (1982).

${ }^{45}$ J. E. Hirsch and E. Fradkin, Phys. Rev. Lett. 49, 402 (1982); E. Frandkin and J. E. Hirsch, Phys. Rev. B 27, 1680 (1983); J. E. Hirsch and E. Fradkin, ibid. 27, 4302 (1983).

${ }^{46}$ H. De Raedt and A. Lagendijk, Phys. Rev. Lett. 49, 1522 (1982); Phys. Rev. B 27, 6097 (1983); 30, 1671 (1984); Phys. Rep. 127, 234 (1985).

${ }^{47}$ C. Alexandrou and R. Rosenfelder, Phys. Rep. 215, 1 (1992), and references therein.

${ }^{48}$ P. E. Kornilovitch, Phys. Rev. Lett. 81, 5382 (1998); Phys. Rev. B 60, 3237 (1999).

${ }^{49}$ M. Hohenadler, H. G. Evertz, and W. von der Linden, Phys. Rev.
B 69, 024301 (2004).

${ }^{50}$ A. Macridin, G. A. Sawatzky, and M. Jarrell, Phys. Rev. B 69, 245111 (2004).

${ }^{51}$ W. Stephan, Phys. Rev. B 54, 8981 (1996); M. Capone, W. Stephan, and M. Grilli, ibid. 56, 4484 (1997).

${ }^{52}$ E. Jeckelmann and S. R. White, Phys. Rev. B 57, 6376 (1998); E. Jeckelmann, C. Zhang, and S. R. White, ibid. 60, 7950 (1999); C. Zhang, E. Jeckelmann, and S. R. White, ibid. 60, 14092 (1999).

${ }^{53}$ T. Holstein, Ann. Phys. (N.Y.) 8, 325 (1959); Ann. Phys. (N.Y.) 8, 343 (1959).

${ }^{54}$ The Holstein polaron is lighter than the Fröhlich polaron in the weak coupling regime (Ref. 36), and also if lattice vibrations are polarized along the hopping direction in the strong-coupling regime [see in S. A. Trugman, J. Bonča, and L.-C. Ku, Int. J. Mod. Phys. B 15, 2707 (2001)].

${ }^{55}$ H. Fröhlich, Adv. Phys. 3, 325 (1954).

${ }^{56}$ H. Fehske, J. Loos, and G. Wellein, Phys. Rev. B 61, 8016 (2000).

${ }^{57}$ A. S. Alexandrov and C. Sricheewin, Europhys. Lett. 51, 188 (2000).

${ }^{58}$ J. Bonča and S. A. Trugman, Phys. Rev. B 64, 094507 (2001).

${ }^{59}$ A. S. Alexandrov and P. E. Kornilovitch, J. Phys.: Condens. Matter 14, 5337 (2002).

${ }^{60}$ D. M. Eagles, Phys. Rev. 130, 1381 (1963).

${ }^{61}$ B. B. Beard and U. J. Wiese, Phys. Rev. Lett. 77, 5130 (1996).

${ }^{62}$ R. P. Feynman, Statistical Mechanics (Benjamin, Reading, MA, 1972).

${ }^{63}$ K. Binder and D. W. Heermann, Monte Carlo Simulation in Statistical Physics: An Introduction (Springer, Berlin, 1988).

${ }^{64}$ N. Metropolis, A. N. Rosenbluth, M. N. Rosenbluth, A. H. Teller, and E. Teller, J. Chem. Phys. 21, 1087 (1953).

${ }^{65}$ E. Maxwell, Phys. Rev. 78, 477 (1950).

${ }^{66}$ C. A. Reynolds, B. Serin, W. H. Wright, and N. Nesbitt, Phys. Rev. 78, 487 (1950).

${ }^{67}$ H. Fröhlich, H. Pelzer, and S. Zienau, Philos. Mag. 41, 221 (1950).

${ }^{68}$ S. I. Pekar, Zh. Eksp. Teor. Fiz. 19, 796 (1949).

${ }^{69}$ G.-M. Zhao, K. Conder, H. Keller, and K. A. Müller, J. Phys.: Condens. Matter 10, 40 (1999).

${ }^{70}$ F. Marsiglio, in Recent Progress in Many-Body Theories, Vol. 4 edited by E. Schachinger, H. Mitter, and H. Sormann (Plenum, New York, 1995).

${ }^{71}$ P. E. Kornilovitch and A. S. Alexandrov, Phys. Rev. B 70, 224511 (2004) 